\title{
ON $p$-SOLUBLE GROUPS AND THE NUMBER OF SIMPLE MODULES ASSOCIATED WITH A GIVEN BRAUER PAIR
}

\author{
By LAURENCE BARKER
}

[Received 6 May 1996; in revised form 20 July 1996]

\section{Statement and discussion of the main result}

THROUGHOUT, we fix an algebraically closed field $k$ of prime characteristic $p$, and a finite group $G$. All the results stated in this section will be proved in Section 5.

TheOREM 1.1. Suppose that $G$ is p-soluble, let $P$ be a $p$-subgroup of $G$, and let $b$ be a block of $k N_{G}(P)$. Then the number of isomorphism classes of simple $k N_{G}(P) b$-modules with vertex $P$ equals the number of isomorphism classes of simple $k G$-modules $X$ with vertex $P$ such that $b$ fixes the Green correspondent of $X$.

Theorem 1.1 further refines Okuyama's refinement, Corollary 1.3 below, of the $p$-soluble case of the block form of Alperin's (weight) conjecture. More to the point, Theorem 1.2 below, a reformulation of Theorem 1.1, tells us that when $G$ is $p$-soluble, the number of isomorphism classes of simple $k G$-modules associated with a given self-centralising Brauer pair is a local invariant.

Recall that Sibley [28] assigned, to any indecomposable $k G$-module $X$, a Brauer pair, well-defined up to $G$-conjugation, called a vertex pair of $X$. The vertex pairs of any simple $k G$-module are self-centralizing.

TheOREM 1.2. Suppose that $G$ is p-soluble, and let $(P, \omega)$ be a self-centralizing Brauer pair on $k G$. Then the number of isomorphism classes of simple $k G$-modules with vertex pair $(P, w)$ equals the number of isomorphism classes of simple $k N_{G}(P)$-modules with vertex pair $(P, w)$.

The conclusions of Theorems 1.1 and 1.2 are both false for arbitrary $G$. If, however, instead of assuming $G$ to be $p$-soluble, we assume $G$ to have abelian Sylow p-subgroups, it is easy to show, using Knörr [11, 3.7], that the conclusions of Theorems 1.1 and 1.2 are both equivalent to the assertion that the block form of Alperin's conjecture [1, page 371] holds for $G$. Theorem 1.2 may indicate some virtue in seeking generalisations of Alperin's conjecture in terms of self-centralizing Brauer pairs. In Puig's letter [25], he suggests such a generalisation, and shows that it is susceptible to the "inversion" techniques in Knörr-Robinson [12] (suitably adapted for the $G$-poset of Brauer pairs). These remarks, together 
with the Kulshammer-Puig generalisation $[13,1.20 .3]$ of Dade's theorem $[6,10]$ perhaps suggest the possibility of extending the machinery in this paper to reduce, to the almost simple cases, local assertions relating to Alperin's conjecture.

CoROLlary 1.3. (Okuyama) Suppose that $G$ is p-soluble.

(a) Let $P$ be a p-subgroup of $G$, and let a be a block of $k G$. Then the number of isomorphism classes of simple $k G a$-modules with vertex $P$ equals the number of isomorphism classes of simple $k N_{G}(P)$ modules $Y$ with vertex $P$ such that a fixes the Green correspondent of $Y$.

(b) In particular, the block form of Alperin's conjecture holds for $G$.

Manz and Wolf's remark [16, page 284] “. .. it has been widely rumored for many years that Okayama has verified the weight conjecture for p-solvable groups..." only hints at the extent to which the result-its proof and the history of its proof-has been shrouded in mystery. Alperin [1, page 371] states Corollary 1.3(b), citing Okuyama [19], but $[19,4.1]$ is actually the special case of Corollary 1.3(a) where $P$ is a defect group of a. Corollary 1.3(a) is Okuyama [18, Theorem 5] (reference specifications indicate this undated typescript to have been written in 1980 or 1981). The last line of Okuyama's argument elides over the need to check that Dade's equivalence $[6,10]$ can be chosen compatibly with the Brauer correspondence. However, Puig's improvement [23, (e)] of Dade [6, 10] establishes this compatibility property; see also Theorem 4.1. Corollary 1.3(b) has a sketch proof in Robinson-Staszewski [27, page 241], and appears as Gres' $[9,2.17]$. In both works, Okuyama's omission is repeated, and in [9], an errror is introduced. The rider to Corollary 1.4 below, which was communicated to me by Isaacs, shows that the first part of $\left[9,2.1\right.$ (ii)] is false; the non-trivial 2-weight of $S_{4}$ provides an obvious counter-example to the second part of $[9,2.1$ (ii)].

The argument in Okuyama [18] can also be interpreted (without invoking Puig [23]) as a proof that if $G$ is $p$-soluble with $p$-subgroup $P$, then the number of isomorphism classes of simple $k G$-modules with vertex $P$ equals the number of isomorphism classes of simple $k N_{G}(P)$ modules with vertex $P$. Isaacs-Navarro [10, Theorem A] generalises this result. Dade [8] announces that a generalisation of Alperin's conjecture can be reduced to the case of an almost simple group, and he informs me that his reduction subsumes a proof of Corollary 1.3(b). Puig's manuscript [26], considerably more complicated than this paper but, equally, more profound, obtains a bijection $\chi_{\omega, P, G}$ from the isomorphism classes of simple $k G$-modules with vertex $P$ to the isomorphism classes of simple $k N_{G}(P)$-modules with vertex $P$. Here, the parameter $\omega$ (independent of $P$ and $G$ ) is a construction based on the Dade groups of the finite 
p-groups. Puig informs me of the following: for fixed $\omega$, and varying $P$, $G$, the bijections $\chi_{\omega, P, G}$ are natural (in the sense that they commute with group isomorphisms); $\omega$ may be chosen canonically when $|G|$ is add or $p=2$; for each $\omega, P, G$, the bijection $\chi_{\omega, P, G}$ is compatible with blocks in such a way that Corollary $1.3(\mathrm{a})$ is a consequence, and $\chi_{\omega, P, G}$ is compatible with defect pairs (defined below), thus giving another proof of Theorems 1.1 and 1.2 .

COROLlary 1.4. Suppose that $G$ is p-soluble, and let $P$ be a p-subgroup of $G$. Then the following two conditions are equivalent:

(1) There exist two simple $k N_{G}(P)$-modules with vertex $P$ in distinct blocks of $k N_{G}(P)$ but with Green correspondents in the same block of $k G$.

(2) There exist two simple $k G$-modules with vertex $P$ in the same block of $k G$ but with Green correspondents in distinct blocks of $k N_{G}(P)$.

Moreover, when $p=2$, there exists a finite soluble group $G$ with $a$ 2-subgroup $P$ satisfying these conditions.

If $G$ is $p$-soluble, and $X$ is a simple $k G$-module with vertex $P$ and source $k P$-module $S$, then the isomorphism class of $S$ is $N_{G}(P)$-stable, and the twisted group algebra associated with a defect multiplicity module of $X$ splits. That is to say, in the notation of Section 2, there is an equivalence $\tilde{\eta}: k_{\sharp} N_{G}(P, S) \neg k N_{G}(P)$ of twisted group algebras over $N_{G}(P)$. This discovery, due to Puig, is an immediate consequence of Kulshammer-Puig [13, 2.12.4] and Puig's result in Thévenaz [29, 30.5]. The only use we make, in this paper, of the existence of $\tilde{\eta}$ is the following illustration of a problem which we shall have to face in Section 4. The defect multiplicity $k_{\sharp} N_{G}(P, S)$-module $V_{G}(P, S)$ of $X$, being the inflation of a simple projective $k_{\sharp} \bar{N}_{G}(P, S)$-module (see Section 2), may be regarded, via $\tilde{\eta}$, as a simple $k N_{G}(P)$-module with vertex $P$. Unfortunately, it is unclear whether or not it is possible thus or otherwise to obtain, for all $p$-soluble $G$, a canonical bijection from the isomorphism classes of simple $k N_{G}(P)$-modules with vertex $P$. No canonical choice of $\tilde{\eta}$ is known. Navarro [17, Theorem B] exhibits such a canonical bijection when $|G|$ is odd. Puig informs me that $\tilde{\eta}$ may be chosen canonically when $|G|$ is odd or $p=2$.

Another problem (more precisely, another manifestation of the same problem) arises in connection with Dade's theorem $[6,10]$ (proved in the unpublished typescript Dade [7, 9.1], and also in Puig [23, (e)]), which asserts the existence of an equivalence of twisted group algebras

$$
\text { v: } k_{\#} N_{G}(P) / C_{R}(P) \cong k_{\sharp} N G(P) R / R
$$

via the canonical group isomorphism $N_{G}(P) / C_{R}(P) \cong N_{G}(P) R / R$. Here, $G$ is arbitrary with $p$-subgroup $P$ and normal $p^{\prime}$-subgroup $R$, and the two 
twisted group algebras involved are defined in terms of a $G$-stable block $e$ of $k R$ such that $k G e$ has an indecomposable module with vertex $P$; see Theorem 4.1 for details. Dade mistakenly stated in [6] that $\tilde{v}$ is canonical, but he corrected this in [7], giving an example to show that "there can be several [equivalences]...each just as natural as the others, with no obvious way to choose one among them in a suitable functorial fashion." (Note that Dade's correction underlines the need, in Okuyama's proof of Corollary 1.3(a), to check that $\tilde{v}$ may be chosen compatibly with the Brauer correspondence.)

Both of these problems (or rather, this single essential problem) may be circumvented by restricting to $C_{G}(P)$. More precisely, it is well-known (see Section 2) that for $G$ arbitrary, and for any indecomposable $k G$-module $X$ with vertex $P$ and source $k P$-module $S$, the preimage of $C_{G}(P)$ in $k_{\sharp} N_{G}(P, S)$ splits canonically. The defect multiplicity module $V_{s}(X, S)$ thus restricts to a (well-defined) $k C_{G}(P)$-module. Also, by Puig $[23,(\mathrm{e})]$ (see Theorem 4.1), $\tilde{v}$ may be chosen so as to restrict to a canonical equivalence

$$
\tilde{v}_{C}: k_{\sharp} C_{G}(P) / C_{R}(P) \cong k_{\sharp} C_{G}(P) R / R
$$

and moreover, $\tilde{v}_{C}$ is compatible with the Brauer correspondence.

The preparatory material in Section 2, and the reviews, at the beginnings of Sections 3 and 4, of Fong's correspondences, may strike some as pedantic. However, we have good reason for paying careful attention to detail. Initial work on refining the $p$-soluble case of Alperin's conjecture, begun with Puig in 1992 (see ACKNOWLEDGEMENTS), was beset by a difficulty in keeping track of equivalences of twisted group algebras. The different approaches of Puig and Thévenaz to the parametrisation of primitive interior $G$-algebras-see the references cited in Thévenaz [29, Section 26]-reveal this difficulty to run deep in $G$-algebra theory. It may be illuminating to note that the result $[3,8.3]$, although intended specifically for strengthening Corollary 1.3(a), transpired to be of no help towards this objective because, at the time [3] was written, I had not understood the need to establish more than the mere existence of the implicit equivalence of twisted group algebras. Rather than entangling ourselves in the intricacies of this equivalence itself, which is probably non-canonical anyway, our policy of restricting to the centralizer $C_{G}(P)$ of the $p$-subgroup $P$ concerned "cuts the Gordian knot" by replacing the original equivalence with a canonical and more tractable one which is eventually realised, in the proof of Proposition 4.8, after making suitable identifications, as simply the identity equivalence $k C_{G}(P) \cong k C_{G}(P)$. Nervertheless, to achieve this realisation, relationships between other twisted group algebras of $C_{G}(P)$ will need to be examined, and the two above-mentioned problems (the single essential 
problem) will be present in the background. It seems worth making an effort to avoid eliding over these subtleties which, perhaps, I am not alone in having been confused by.

Any primitive interior $G$-algebra $A$ (over $k$ or over a suitable discrete valuation ring) may be assigned a pair $(P, W)$, well-defined up to $G$-conjugacy, called a defect pair of $A$, where $P$ is a $p$-subgroup of $G$, and $W$ is a simple $k C_{G}(P)$-module. Defect pairs will be more thoroughly investigated in [4]. In Section 2, we shall discuss defect pairs only in the context of $k G$-modules. Generally, the notion of a defect pair is a refinement of that of a vertex pair. However, of particular significance for our purposes here, given a simple $k G$-module $X$ with vertex $P$ and source $k P$-module $S$, the defect pairs and vertex pairs of $X$ (that is, of $\operatorname{End}_{k}(X)$ ) are interchangeable, and are determined by the restriction to $k C_{G}(P)$ of the defect multiplicity module $V_{G}(X, S)$. Shifting attention from vertex pairs to defect pairs will not increase the information at our disposal, but it will allow us to make use of module-theoretic operations such as inflation, induction, and Morita equivalence, which would be awkward to express in terms of vertex pairs.

$G$-Algebra theory, while extending the local theory of Brauer and Green, also encapsulates much of Clifford theory, and is conducive to expressing, in a manner easily susceptible to local reduction, the Morita equivalences inherent in Fong reduction. The use of $G$-algebra theory to elucidate Fong reduction, well-known to some but not already explicit in the literature, has its origins in Cliff [5] and Puig [21]. Fong reduction, recall, is applicable in the presence of a normal $p^{\prime}$-subgroup $R$ of $G$, and a block $e$ of $k R$. We shall decompose Fong reduction into two parts: Fong's first correspondence, reducing to the case where $e$ is $G$-stable, and Fong's second correspondence, replacing $R$ with a central cyclic $p^{\prime}$ subgroup. In Section 3, we investigate the behaviours, under Fong's first correspondence, of defect multiplicity modules and defect pairs of indecomposable modules. Section 4 is partially analogous, first reinterpreting the construction in Puig [23] of Dade's equivalence (denoted $\bar{v}$ above), then showing how Fong's second correspondence respects the defect multiplicity modules and defect pairs of a fairly broad class of indecomposable modules, namely, the simply defect indecomposable modules (which, defined in Section 2, include the simple modules). Our main concern, in this work, is with simple modules, but the wider scope incurs little extra hardship, and allows Sections 2 and 3 to attempt a general exposition of a fundamental connection between $G$-algebra theory and some traditional Clifford theory.

The general theoretical material having been developed, we shall then be ready, in Section 5, to demonstrate all the results stated in this section. The main effort will be to prove Theorem 1.2, reformulated in terms of 
defect pairs. It is interesting to note that if, in the inductive hypothesis, the conclusion of Corollary 1.3(a) is substituted for the conclusion of Theorem 1.2, then the argument falters at the application of Fong's first correspondence (Step 5.2). The reason for this hitch is explained in the second paragraph of Section 3. Okuyama [18] circumvents this hitch by using different induction parameters, and some group-theoretic reasoning. We might speculate, however, that an attempt to adapt results in this section to situations where fewer group-theoretic constraints are available could perhaps benefit from the more ring-theoretic approach effected by the notion of a defect pair.

\section{Multiplicity modules and defect pairs}

Recall that J. A. Green defined a $G$-algebra over $k$ to be an algebra over $k$ upon which $G$ acts as automorphisms. To comprehend the technical manoeuvres in subsequent sections, a little familiarity with Puig's theory of $G$-algebras, as established in, for instance, Puig [22], [24] will be advantageous. Thévenaz [29, Chapters $2,3,6]$ gives a more leisurely exposition of all the relevant $G$-algebra theory. In this section, we given an account of some very well-known theory of twisted group algebras, and then some fundamental $G$-algebra theory in the easy special case which concerns us, that of $k G$-modules and their endomorphism algebras. The presentation below of the constructions we require is more-or-less self-contained except in that we shall refer to other sources for some proofs. Our justification for devoting space to a simplification of material already in the literature is not only to set notation in a way which will turn out to be convenient later, but more importantly, to introduce some terminology enabling us, in subsequent sections, to discuss equivalences of twisted group algebras precisely. Indeed, the concept of an $R$-normal twisted group algebra of $G$ (see below), and the use of this concept when dealing with defect multiplicity modules or with Fong's second correspondence are crucial to the way we examine, in Section 4, the effect of Fong's second correspondence on defect multiplicity modules. The only novelty in this section is the notion of a defect pair, but we shall demonstrate only a few properties of defect pairs here, postponing more thorough discussion to [4].

Let us begin with some generalities on twisted group algebras. Given an algebra $A$ over $k$ (all algebras deemed finite-dimensional), we write $A^{*}$ for the group of units of $A$. We take the view that a twisted group algebra of $G$ (over $k$ ) is an algebra $k_{*} G$ over $k$ equipped with a $k$-linear decomposition $k_{\sharp} G=\bigoplus_{8 \in G}\left(k_{*} G\right)_{g}$ such that each vector space $\left(k_{*} G\right)_{g}$ is 
1-dimensional, and $\left(k_{\sharp} G\right)_{g}\left(k_{\sharp} G\right)_{h}=\left(k_{\sharp} G\right)_{g h}$ for all $g, h \in G$. Let us fix twisted group algebras $k_{\sharp} G$ and $k_{\#} G^{\prime}$ of $G$. We say that $k_{\sharp} G$ and $k_{\sharp} G^{\prime}$ are equivalent provided there exists an equivalence $k_{\sharp} G \cong k_{\sharp} G^{\prime}$, that is, an algebra isomorphism $k_{\sharp} G \cong k_{\sharp} G^{\prime}$ which restricts, for each $g \in G$, to a $k$-linear isomorphism $\left(k_{\sharp} G\right)_{g} \cong\left(k_{\sharp} G^{\prime}\right)_{g}$. (We avoid the usual term "isomorphism" in place of "equivalence" because any non-trivial automorphism of $G$ induces an isomorphism $k G \cong k G$ in the category of twisted group algebras of finite groups, but this isomorphism is not an equivalence.) We define a proper basis for $k G$ to be a basis of the form $\tilde{G}=\{\tilde{g}: g \in G\}$ such that each $\tilde{g} \in\left(k_{\#} G\right)_{g}$. Given such a proper basis $\widetilde{G}$, writing $\alpha$ for the factor set $G \times G \rightarrow k^{*}$ given by $\tilde{g} \tilde{h}=\alpha(g, h) \widetilde{g h}$, and writing $\left[k_{\sharp} G\right]$ for the image of $\alpha$ in the second-degree cohomology group $H^{2}\left(G, k^{*}\right)$ (where $G$ acts trivially on $k^{*}$ ), it is well-known that $\left[k_{\sharp} G\right]$ is independent of the choice of proper basis $G$, and that $\left[k_{\sharp} G\right]$ uniquely determines $k_{\#} G$ up to equivalence. The subgroup $\Delta\left(k_{\#} G\right)$ of $\left(k_{*} G\right)^{*}$ generated by $k^{*}$ and $G$ is also independent of the choice of $\bar{G}$, and $k_{\sharp} G$ is canonically isomorphic to $k \bigotimes_{k\left(k^{*}\right)} k \Delta\left(k_{\sharp} G\right)$.

We define a $k_{\sharp} G$-algebra to be an algebra $A$ over $k$ equipped with a structural map $k_{\sharp} G \rightarrow A$. The interior $G$-algebras are precisely the $k G$-algebras. Any $k_{\sharp} G$-algebra is a $G$-algebra whereby each $g \in G$ acts as conjugation by $\tilde{g}$. All the twisted group algebras used to prove the results in Section 1 will arise in the following way. Given a simple $G$-algebra $S$ over $k$, then by the Noether-Skolem theorem and the fact that $k$ is algebraically closed, the $G$-algebra structure of $S$ lifts to a $k_{*} G^{S}$-algebra structure for some twisted group algebra $k_{\sharp} G^{S}$ of $G$. (We mean that $g$ acts on $S$ as conjugation by any non-zero element of $\left(k_{\sharp} G\right)_{g}$.) Moreover, $S$ determines $k_{\sharp} G^{S}$ up to a unique equivalence; if the $G$-algebra structure of $S$ lifts to a $k_{*} G^{\prime}{ }^{\prime}$-algebra structure for another twisted group algebra $k_{\sharp} G^{\prime}$ of $G$, then there exists a unique equivalence $k_{\sharp} G^{\prime S} \approx k_{\sharp} G^{S}$ commuting with the structural maps $k_{\sharp} G^{\prime s} \rightarrow S$ and $k_{*} G^{S} \rightarrow S$. Indeed, this follows from the uniqueness property of pullbacks because the short exact sequence $1 \rightarrow k^{*} \rightarrow \Delta\left(k_{*} G^{S}\right) \rightarrow G \rightarrow 1$ is determined up to an isomorphism condition by pull-back from the short exact sequence $1 \rightarrow k^{*} \rightarrow$ Aut $(S) \rightarrow S^{*} \rightarrow 1$.

Continuing with the twisted group algebras $k_{\sharp} G, k_{\sharp} G^{\prime}$ of $G$, the twisted group algebra $k_{\sharp} G * k_{\sharp} G^{\prime}$ of $G$ is defined to be the subalgebra $\bigoplus_{g \in G}\left(k_{*} G\right)_{g} \otimes\left(k_{*} G^{\prime}\right)_{g} \quad$ of $\quad k_{\#} G \otimes k_{*} G^{\prime} \quad$ with $\quad\left(k_{*} G * k_{*} G^{\prime}\right)_{g}=$ $\left(k_{\#} G\right)_{g} \otimes\left(k_{\#} G^{\prime}\right)_{g}$. (All our tensor products, unless otherwise indicated, are over $k$.) It is easy to check that $\left[k_{*} G * k_{*} G^{\prime}\right]=\left[k_{*} G\right]+\left[k_{*} G^{\prime}\right]$. Given modules $V, V^{\prime}$ of $k_{*} G, k_{*} G^{\prime}$, respectively, then $V \otimes V^{\prime}$ is evidently a $k_{\#} G * k_{\sharp} G^{\prime}$-module. Likewise, given a $k_{\sharp} G$-algebra $A$, and a $k_{\sharp} G^{\prime}$-algebra $A^{\prime}$, then $A \otimes A^{\prime}$ is a $k_{\sharp} G * k_{*} G^{\prime}$-algebra. 
We define the opposite twisted group algebra of $k_{*} G$ to be the twisted group algebra $k_{\sharp} G^{o}$ of $G$ such that $k_{\sharp} G$ and $k_{\sharp} G^{o}$ have the same underlying rings, $\left(k_{\sharp} G\right)_{g}=\left(k_{\sharp} G^{o}\right)_{g}$ as sets for each $g \in G$, and the vector space structures of $k_{\sharp} G$ and $k_{\sharp} G^{o}$ are related by the condition that the action of any element $\kappa \in k^{*}$ on $k_{\sharp} G$ coincides with the action of $\kappa^{-1}$ on $k_{\sharp} G^{o}$. Given a proper basis $\tilde{G}=\{\tilde{g}: g \in G\}$ of $k_{\sharp} G$, we define the opposite basis of $\tilde{G}$ to be the proper basis $\tilde{G}^{o}=\left\{\tilde{g}^{o}: g \in G\right\}$ such that each $\tilde{g}^{o}$ coincides with $\tilde{g}$ as an element of the underlying ring. The two factor sets $G \times G \rightarrow k^{*}$ associated with $\bar{G}$ and $G^{\circ}$ commute with inversion in $k^{*}$. For any function $\lambda: G \rightarrow k^{*}$, the proper basis $\{\lambda(G) \bar{g}: g \in G\}$ has opposite basis $\left\{\lambda(G)^{-1}: g \in G\right\}$. From this, it is easy to see that there is a canonical equivalence $k G \cong k_{\sharp} G * k_{*} G^{\circ}$ whereby each $g \in G$, regarded as an element of $k G$, corresponds to $\tilde{g} \otimes \tilde{g}^{o}$. If there exists an equivalence $\tilde{\mu}: k_{\sharp} G \neg k_{\sharp} G^{\prime}$, then we define the opposite equivalence of $\tilde{\mu}$ to be the equivalence $\tilde{\mu}^{o}: k_{\sharp} G^{o} \simeq k_{\sharp} G^{\prime o}$ such that $\tilde{\mu}$ and $\tilde{\mu}^{o}$ coincide as ring isomorphisms. The following lemma is a special case of Puig's result in $[3,8.2]$. We restate it to be clear about the equivalences involved, but the proof remains unchanged.

Lemma 21 (Puig). Let $A$ be an interior $G$-algebra, let $S$ be a $G$-stable simple subalgebra of $A$ such that $1_{S}=1_{A}$, let $C$ be the centralizer of $S$ in $A$, and let $k_{*} G$ be a twisted group algebra of $G$ such that the $G$-algebra structure of $S$ lifts to a $k_{\sharp} G$-algebra structure. Then the $G$-algebra structure of $C$ lifts to a $k_{\#} G^{o}$-algebra structure in a unique way such that, via the canonical equivalence $k G \cong k_{\sharp} G * k_{\sharp} G^{o}$, we have an interior $G$-algebra isomorphism $A \cong S \otimes C$ given by $s c \leftrightarrow s \otimes c$ for $s \in S$ and $c \in C$.

Given a subgroup $H \leqslant G$, the subalgebra $k_{\#} H:=\bigoplus_{f \in H}\left(k_{\#} G\right)_{f}$ of $k_{\#} G$ is evidently a twisted group algebra of $H$. We call $k_{\sharp} H$ the preimage of $H$ in $k_{\sharp} G$. For a $k_{\sharp} G$-module $X$, and a $k_{\sharp} H$-module $Y$, we write $\operatorname{Res}_{H}^{G}(X)$ for the restriction of $X$ to $k_{\sharp} H$, and write $\operatorname{Ind}_{H}^{G}(Y)$ for the induced $k_{\#} G$-module $k_{\#} G \bigotimes_{k_{H} H} Y$.

Let $R$ be an normal subgroup of $G$, let $G:=G / R$, and for each $g \in G$, let $g$ denote the image of $g$ in $G$. Writing $k_{*} R$ for the preimage of $R$ in $k_{\sharp} G$, let us suppose that the twisted group algebra $k_{\sharp} G$ is equipped with an equivalence $\bar{v}: k R \neg k_{\#} R$ such that $\bar{v}(R)$ is normal in $\Delta\left(k_{z} G\right)$. Then we call $k_{\sharp} G$ an $R$-normal twisted group algebra of $G$. We identify $k R$ with $k_{\sharp} R$ via $\tilde{v}$, and in particular, write $\tilde{v}(x)=x$ for $x \in R$. We define an $R$-normal basis for $k_{\sharp} G$ to be a proper basis $\{\tilde{g}: G \in G\}$ such that $x=\tilde{x}$ and $x \tilde{g}=\widetilde{x g}$ for all $x \in R$ and $g \in G$. Then the identification of $k R$ with $k_{\sharp} R$ allows us to regard $R$ as a normal subgroup of $\Delta\left(k_{\sharp} G\right)$, whereupon the conjugation actions of $g$ and of $\tilde{g}$ on $R$ conicide, and $\tilde{g} x=\tilde{g} x \tilde{g}^{-1} \tilde{g}=$ 
$g x g^{-1} \tilde{g}=\widetilde{g x}$. Suppose that $k_{\sharp} G^{\prime}$ is another $R$-normal twisted group algebra of $G$. We define an $R$-nomgl equivalence $k_{*} G \neg k_{t} G^{\prime}$ to be an equivalence $k_{\#} G \neg k_{\#} G^{\prime}$ which restricts to the identity automorphism of $k R$. Clearly, given an $R$-normal equivalence $\bar{\mu}: k_{\sharp} G \Im k_{\#} G^{\prime}$, then $\tilde{\mu}^{o}: k_{\sharp} G^{o} \Im k_{\sharp} G^{o}$ is also $R$-normal.

The following cautionary example should make it clear that the notion of an $R$-normal twisted group algebra of $G$ is not just a reinvention of a "graded Clifford system" or a "crossed product". Suppose that $p$ is odd, let $G:=D_{8} / Z\left(D_{8}\right)$, and let $R$ be the subgroup of $G$ generated by the image of a reflection. Let $k_{\sharp} G:=k \underset{k Z\left(D_{4}\right)}{\otimes_{8}} k D_{8}$, where the central involution in $D_{8}$ acts on $k$ as negation. Despite $k_{\#} R$ being equivalent to $k R$, it is impossible to given $k_{\#} G$ an $R$-normal structure.

To indicate the use we shall make of $R$-normal twisted group algebras of $G$, suppose that the simple $G$-algebra $S$, as above, is also an interior $R$-algebra in such a way that the action of $R$ by conjugation coincides with the restriction to $R$ of the action of $G$. By the pull-back construction of $\Delta\left(k_{\#} G^{S}\right)$, there exists a unique equivalence $\tilde{\nabla}: k R \neg R^{S}$ such that $\theta_{R}=\theta_{G} \tilde{v}$, where $k_{\sharp} R^{S}$ is the preimage of $R$ in $k_{\sharp} G^{S}$, and $\theta_{R}: k R \rightarrow S$ and $\theta_{G}: k_{*} G^{S} \rightarrow S$ are the structural maps. Given a non-zero element $\tilde{g} \in\left(k_{*} G^{S}\right)_{g}$, and $x \in R$, then $\tilde{g} \tilde{v}(x) \tilde{g}^{-1}$ is clearly a $k^{*}$-multiple of $\tilde{v}\left({ }^{8} x\right)$. But $\theta_{G}\left(\tilde{g} \tilde{v}(x) \tilde{g}^{-1}\right)={ }^{8} \theta_{R}(x)=\theta_{G}\left(\tilde{v}\left({ }^{8} x\right)\right)$, so $\tilde{g} \tilde{v}(x) \tilde{g}^{-1}=\tilde{v}\left({ }^{8} x\right)$. Therefore $\tilde{v}(R)$ is normal in $\Delta\left(k_{\sharp} G^{S}\right)$. We have shown that $k_{\sharp} G^{S}$, equipped with $\bar{v}$, is $R$-normal.

Still supposing $k_{\sharp} G$ to be an $R$-normal twisted group algebra of $G$, let $k_{\sharp} G$ be a twisted group algebra of $G$, and let $\theta$ be an algebra epimorphism $k_{\sharp} G \rightarrow k_{\sharp} G$ such that $\theta\left(\left(k_{v} G\right)_{g}\right)=\left(k_{\sharp} G\right)_{g}$ and $\theta(x)=1$ for all $g \in G$ and $x \in R$. Then we call $k_{\sharp} G$ an inflation of $k_{*} G$ to an $R$ normal twisted group algebra of $G$. We regard $k_{\sharp} G$ as a $k_{\sharp} G$-algebra via $\theta$. The following facts are easy to check. The structural map $\theta$ provides a bijective correspondence between the $R$-normal bases $\bar{G}=\{\tilde{g}: g \in G\}$ of $k_{\#} G$, and the proper bases $\tilde{G}=\{\tilde{g}: g \in G\}$ of $k_{\#} G$ whereby $G \leftrightarrow G$ provided each $\theta(\tilde{g})=\xi$. When $G$ and $\bar{G}$ thus correspond, we call $\tilde{G}$ the lift of $\bar{G}$ to $k_{\sharp} G$. Then the factor sets $\alpha: G \times G \rightarrow k^{*}$ and $\alpha: G \times G \rightarrow$ $k^{*}$ associated with $\bar{G}$ and $\bar{G}$ determine each other by the condition that $\alpha(g, h)=\underline{\alpha}(\underline{g}, \underline{h})$ for all $g, h \in G$. Any twisted group algebra of $\underline{G}$ inflates to an $R$-normal twisted group algebra of $G$, and any $R$-normal twisted group algebra of $G$ is an inflation of a twisted group algebra of $G$. Furthermore, given another twisted group algebra $k_{\sharp} G^{\prime}$ of $G$, supposing $k_{*} G^{\prime}$ to be an inflation of $k_{*} G^{\prime}$ to an $R$-normal twisted group algebra of $G$, and letting $\theta^{\prime}$ be the structural map $k_{\sharp} G^{\prime} \rightarrow k_{\sharp} G^{\prime}$, then there is a bijective correspondence between the $R$-normal equivalences $\tilde{\mu}: k_{\sharp} G \neg$ $k_{\sharp} G^{\prime}$ and the equivalences $\underline{\mu}: k_{\sharp} G \rightrightarrows k_{\sharp} G^{\prime}$ whereby $\tilde{\mu} \leftrightarrow \mu$ provided 
$\theta^{\prime} \tilde{\mu}=\mu \theta$. Thus the equivalence class of a twisted group algebra of $\underline{G}$ uniquely determines the $R$-normal equivalence class of its inflations.

Let us turn now to some $G$-algebra theory, particularly representation theory of $k G$. Given a subgroup $H \leqslant G$, we define $\bar{N}_{G}(H):=N_{G}(H) / H$. Given a $G$-algebra $A$ over $k$, we write $\operatorname{Tr}_{H}^{G}$ for the relative trace map $A^{H} \rightarrow A^{G}$, and define $A_{H}^{G}:=\operatorname{Tr}_{H}^{G}\left(A^{H}\right)$. For a $p$-subgroup $P \leqslant G$, we write $\mathrm{Br}_{\rho}^{G}$ for the Brauer map $(k G)^{P} \rightarrow k C_{G}(P)$. The following version of Nagao's theorem is immediate from Landrock [15, III.1.12, III.5.1].

Theorem 2.2 (Nagao). Let e be a central idempotent of $k G$, let $P$ be a $p$-subgroup of $G$, and let $c:=\mathrm{Br}_{p}^{G}(e)$. Then $c \neq 0$ if and only if there exists an indecomposable $k G e-$ module with vertex $P$. When these equivalent conditions hold, $c$ is a central idempotent of $k N_{G}(P)$, and the Green correspondence restricts to a bijective correspondence between the isomorphism classes of indecomposable $k G e$-modules with vertex $P$, and the isomorphism classes of indecomposable $k N_{G}(P) c$-modules with vertex $P$.

As in Puig [24] and Thévenaz [29, Chapter 2], a multiplicity module can be constructed for any pointed group on any $G$-algebra. We review this construction in the case of the endomorphism algebra of a $k G$ module, customizing notation for our application, and dispensing with some abstractions. Let $H \leqslant G$, let $U$ be an indecomposable $k H$-module, and let $X$ be any $k G$-module such that $U$ is a direct factor of the restriction $\operatorname{Res}_{H}^{G}(X)$. The linear endomorphism algebra $\operatorname{End}_{k}(X)$ is an interior $G$-algebra whose structural map is the representation of $X$. (It is via this observation that our constructions here are specialisations of those in [24] and [29].) The stabilizer $N_{G}(H, U)$ of $U$ in $N_{G}(H)$ contains $H C_{G}(H)$. The idempotents $i$ of $\operatorname{End}_{k H}(X)$ such that $U \approx i \operatorname{Res}_{H}^{G}(X)$ comprise a conjugacy class $\beta$ of primitive idempotents of $\operatorname{End}_{k H}(X)$. Let $\mu_{\beta}$ be the maximal ideal of $\operatorname{End}_{k H}(X)$ not containing $\beta$, let $\Pi_{G}(X, U)$ be the quotient algebra $\operatorname{End}_{k H}(X) / \mu_{\beta}$, and let $s_{X, U}$ be the canonical projection $\operatorname{End}_{k H}(X) \rightarrow \Pi_{G}(X, U)$. Since $N_{G}(H, U)$ stabilizes $\mu_{\beta}$, we may regard $\Pi_{G}(X, U)$ as an $N_{G}(H, U)$-algebra by insisting that $s_{X, U}$ is an $N_{G}(H, U)$-algebra epimorphism. Now $\Pi_{G}(X, U)$ is a simple algebra, so there exists a twisted group algebra $k_{*} N_{G}(H, U)$ of $N_{G}(H, U)$ such that the $N_{G}(H, U)$-algebra structure of $\Pi_{G}(X, U)$ lifts to a $k_{*} N_{G}(H, U)$ algebra structure. Let $\theta_{X, U}$ denote the structural map $k_{\sharp} N_{G}(H, U) \rightarrow$ $\Pi_{G}(X, U)$. We define the multiplicity module $V_{G}(X, U)$ of $U$ in $X$ to be the $k_{\sharp} N_{G}(H, U)$-module obtained by restricting a simple $\Pi_{G}(X, U)$ module via $\theta_{X, U}$. The action of $\Pi_{G}(X, U)$ on $V_{G}(X, U)$ engenders an identification of $\Pi_{G}(X, U)$ with $\operatorname{End}_{k}\left(V_{G}(X, U)\right)$, and then $\theta_{X, U}$ is the representation of $V_{G}(X, U)$.

The multiplicity module $V_{G}(X, U)$ is well-defined up to isomorphism because $k_{\sharp} N_{G}(H, U)$ is determined up to a unique equivalence. Since an 
equivalence $k_{\sharp} N_{G}(H, U) \cong k_{*} N_{G}(H, U)$ may induce a non-trivial permutation of the isomorphism classes of $k_{\sharp} N_{G}(H, U)$-modules, we emphasise Puig's injunction [24, 5.4] against replacing $k_{\sharp} N_{G}(H, U)$ with its equivalence class.

Given another $k G$-module $X^{\prime}$ such that $U$ is a direct factor of $\operatorname{Res}_{H}^{G}\left(X^{\prime}\right)$, we can regard $\operatorname{End}_{k}(X) \oplus \operatorname{End}_{k}\left(X^{\prime}\right)$ as a subalgebra of $\operatorname{End}_{k}\left(X \oplus X^{\prime}\right)$ with $\operatorname{End}_{k}(X)$ annihilating $X^{\prime}$ and with $\operatorname{End}_{k}\left(X^{\prime}\right)$ annihilating $X$. From this, we see that $k_{*} N_{G}(H, U)$ can be chosen independently of $X$, and such that $V_{G}\left(X \oplus X^{\prime}, U\right) \cong V_{G}(X, U) \oplus$ $V_{G}\left(X^{\prime}, U\right)$. For any $k G$-module $Y$, we can still define $V_{G}(Y, U)$ by taking $V_{G}(Y, U)$ to be the zero $k_{\sharp} N_{G}(H, U)$-module when $U$ is not a direct factor of $\operatorname{Res}_{H}^{G}(Y)$. Given another $k G$-module $Y^{\prime}$, we again have $V_{G}\left(Y \oplus Y^{\prime}, U\right) \cong V_{G}(Y, U) \oplus V_{G}\left(Y^{\prime}, U\right)$. (In fact, Puig has observed that $V_{G}(-, U)$ is an additive functor $\operatorname{Mod}(k G) \rightarrow \operatorname{Mod}\left(k_{\sharp} N_{G}(H, U)\right)$.)

Now $\operatorname{End}_{k H}(X)$ inherits an interior $C_{G}(H)$-algebra structure from $\operatorname{End}_{k}(X)$, and $\Pi_{G}(X, U)$ inherits an interior $C_{G}(H)$-algebra structure from $\operatorname{End}_{k H}(X)$ via $s_{X, U}$. Then $k_{*} N_{G}(H, U)$ becomes a $C_{G}(H)$-normal twisted group algebra of $N_{G}(H, U)$ whereby each $x \in C_{G}(H)$ corresponds to (and will be identified with) the element of $\left(k_{*} N_{G}(H, U)\right)_{x}$ which has the same image as $x$ in $\Pi_{G}(X, U)$. Thus $V_{G}(X, U)$ restricts to a $k C_{G}(H)$-module.

For a $p$-subgroup $P$ of $G$, the inclusion $\bar{C}_{G}(P) \hookrightarrow P C_{G}(P)$ induces a group isomorphism $C_{G}(P) / Z(P) \cong P C_{G}(P) / P$, via which we shall identify $C_{g}(P) / Z(P)$ with $P C_{G}(P) / P$, denoting both of these quotient groups by $\bar{C}_{G}(P)$. It is well-known (see Landrock [15, II.3.9], for instance) that the canonical epimorphism $k C_{G}(P) \rightarrow k \bar{C}_{G}(P)$ induces a bijective correspondence between the blocks of $k C_{G}(P)$ and the blocks of $k \bar{C}_{G}(P)$, and also induces a bijective correspondence between the isomorphism classes of simple $k C_{G}(P)$-modules and the isomorphism classes of simple $k \bar{C}_{G}(P)$ modules. If $D$ is a defect group of a given block of $k C_{G}(P)$, then $D \geqslant Z(P)$, and $D / Z(P)$ is a defect group of the corresponding block of $k \bar{C}_{G}(P)$. Likewise, if $Q$ is a vertex of a given simple $k C_{G}(P)$-module $W$, then $Q / Z(P)$ is a vertex of the simple $k \bar{C}_{G}(P)$-module inflating to $W$. Recall that, for a $p$-subgroup $P$ of $G$, and a block $w$ of $k C_{G}(P)$, we call $(P, w)$ a Brauer pair on $k G$. We say that $(P, w)$ is self-centralizing provided $w$ has defect group $Z(P)$. (Self-centralizing Brauer pairs were the pairs originally introduced by $\mathrm{R}$. Brauer.) Given a simple $k C_{G}(P)$ module $W$, we call $(P, W)$ a local pair on $k G$, and we say that $(P, W)$ is self-centralizing provided $W$ has vertex $Z(P)$. Two local pairs $(P, W)$ and $\left(P^{\prime}, W^{\prime}\right)$ are considered to be the same provided $P=P^{\prime}$ and $W \cong W^{\prime}$. We allow $G$ to permute the Brauer pairs on $k G$ and the local pairs on $k G$ by ${ }^{8}(P, w)=\left({ }^{8} P,{ }^{8} w\right)$ and ${ }^{8}(P, W)=\left({ }^{8} P,{ }^{8} w\right)$ for $g \in G$. By comments above, given a Brauer pair $(P, w)$ and a local pair $(P, W)$ such that $w$ 
fixes $W$, then $(P, w)$ is self-centralizing if and only if $(P, W)$ is self-centralizing, and when these conditions hold, $W$ is the isomorphically unique simple $k C_{G}(P) w$-module. So there is an evident $G$-invariant bijective correspondence between the self-centralizing Brauer pairs on $k G$ and the self-centralizing local pairs on $k G$. (To further motivate the nomenculture, we note that, using a comment in Puig [22, page 267], it is not hard to construct a canonical $G$-invariant bijective correspondence between the local pairs on $k G$ and the local pointed groups on $k G$. In case this is unclear, details will be given in [4].)

Let us now suppose that $X$ is an indecomposable $k G$-module with vertex $P$ and source $k P$-module $S$. Let $\bar{N}_{G}(P, S):=N(P, S) / P$. Given any $z \in Z(P)$, then $\theta_{X, S}(z) \in Z\left(\Pi_{G}(X, S)\right)$. But $z$ is a $p$-element, and $\Pi_{G}(X, S)$ is a simple algebra, so $\theta_{X, S}(z)=1$. Therefore, we may regard $k_{\sharp} N_{G}(P, S)$ as a $P C_{G}(P)$-normal twisted group algebra of $N_{G}(P, S)$ in such a way that $\theta_{X, S}(x)=1$ for all $x \in P$. Hence $k_{\sharp} N_{G}(P, S)$ is $P$-normal, is an inflation of a $\bar{C}_{G}(P)$-normal twisted group algebra $k_{\sharp} \bar{N}_{G}(P, S)$ of $\bar{N}_{G}(P, S)$, and $\Pi_{G}(X, S)$ becomes a $k_{\sharp} \bar{N}_{G}(P, S)$-algebra with structural map $\bar{\theta}_{X, S}$ such that $\theta_{X, S}$ and $\bar{\theta}_{X, S}$ commute with the structural map $k_{\sharp} N_{G}(P, S) \rightarrow k_{\sharp} \bar{N}_{G}(P, S)$. Note that the $k_{\sharp} \bar{N}_{G}(P, S)$-algebra structure of $\Pi_{G}(X, S)$ induces the $\bar{N}_{G}(P, S)$-algebra structure inherited from $\operatorname{End}_{k P}(X)$ via $s_{X, S}$. Let $\bar{V}_{G}(X, S)$ denote the $k_{\sharp} \bar{N}_{G}(P, S)$-module obtained by restricting a simple $\Pi_{G}(X, S)$-module via $\bar{\theta}_{X, s}$. Then $V_{G}(X, S)$ is isomorphic to the inflation of $\bar{V}_{G}(X, S)$ via the structural map of $k_{\sharp} \bar{N}_{G}(P, S)$. By Puig $[24,6.4]$, or Thévenaz $[29,19.3], \bar{V}_{G}(X, S)$ is indecomposable and projective. In particular, the head hd $\left(V_{G}(X, S)\right)$ of $V_{G}(X, S)$ is simple. By Clifford's theorem (suitably adapted in a wellknown way to twisted group algebras),

$$
W_{G}(X, S):=\operatorname{Res}_{C_{G}(P)}^{N_{G}(P)}\left(\operatorname{hd}\left(V_{G}(X, S)\right)\right)
$$

is a direct sum of mutually $N_{G}(P, S)$-conjugate simple $k C_{G}(P)$-modules. Given a simple $k C_{G}(P)$-module $W$, we call $(P, W)$ a defect pair of $X$ provided $W$ occurs in the semisimple $k C_{G}(P)$-module $W_{G}\left(X, S^{\prime}\right)$ for some source $k P$-module $S^{\prime}$ of $X$.

The multiplicity $k_{\sharp} N_{G}(f f, S)$-module $V_{G}(X, S)$ is called a defect multiplicity module of $X$. Let $P^{\prime}$ be another vertex of $X$, and $S^{\prime}$ a source $k P^{\prime}$-module of $X$. As is well-known, there exists an element $g \in G$ such that $P^{\prime}=8 P$ and $S^{\prime} \Xi^{8} S$. The conjugation actions of $g$ on $G$, on $k G$, and on $\operatorname{End}_{k}(X)$ provide, respectively, a group isomorphism

$$
\mu_{\varepsilon}: N_{G}(P, S) \rightrightarrows N_{G}\left(P^{\prime}, S^{\prime}\right),
$$

an algebra isomorphism

$$
\sigma_{\varepsilon}: k C_{G}(P) \rightrightarrows k C_{G}\left(P^{\prime}\right),
$$


and an algebra isomorphism

$$
\rho_{g}: \Pi_{G}(X, S) \subsetneq \Pi_{G}\left(X, S^{\prime}\right) .
$$

Regarding $\Pi_{G}\left(X, S^{\prime}\right)$ as an $N_{G}(P, S)$-algebra via $\mu_{g}$, and as an interior $C_{G}(P)$-algebra via $\sigma_{g}$, then $\rho_{g}$ is both an $N_{G}(P, S)$-algebra isomorphism and an interior $C_{G}(P)$-algebra isomorphism. By the uniqueness of pull-backs, there exists an isomorphism of twisted group algebras $\tilde{\mu}_{g}: k_{*} N_{G}(P, S) \neg k_{\sharp} N_{G}\left(P^{\prime}, S^{\prime}\right)$ inducing $\mu_{g}$, restricting to $\sigma_{g}$, and such that, regarding $\Pi_{G}\left(X, S^{\prime}\right)$ as a $k_{\sharp} N_{G}(P, S)$-algebra via $\bar{\mu}_{g}$, then $\rho_{g}$ is a $k_{\sharp} N_{G}(P, S)$-algebra isomorphism. In particular, $V_{G}(P, S)$ and $V_{G}\left(P^{\prime}, S^{\prime}\right)$ are isomorphic via $\tilde{\mu}_{g}$. This shows a sense in which defect multiplicity modules are unique up to conjugation. It also shows that $W_{G}(X, S)$ is isomorphic to $W_{G}\left(X, S^{\prime}\right)$ via $\sigma_{g}$. So the defect pairs of $X$ comprise a $G$-orbit of local pairs on $k G$.

Let $Y$ be the indecomposable $k N_{G}(P)$-module with vertex $P$ in Green correspondence with $X$. Of course, $Y$ has source $k P$-module $S$, and $N_{G}(P, S)=N_{N_{G}(P)}(P, S)$. Thévenaz [29, 20.1(c)(ii)] describes an isomorphism $\Pi_{G}(X, S) \equiv \Pi_{N_{G}(P)}(Y, S)$ which is both an $N_{G}(P, S)$-algebra isomorphism and an interior $C_{G}(P)$-algebra isomorphism. By the uniqueness of pull-backs, we may identify $k_{*} N_{N_{G}(P)}(P, S)$ with $k_{*} N_{G}(P, S)$ as $C_{G}(P)$-normal twisted group algebras of $N_{G}(P, S)$ in such a way that $V_{N_{G}(P)}(Y, S) \cong V_{G}(X, S)$. Thus, any defect multiplicity module of $Y$ may be regarded as a defect multiplicity module of $X$. It follows that any defect pair of $Y$ is a defect pair of $X$.

After Sibley $[28,2.6]$, for any block $w$ of $k C_{G}(P)$ such that $\theta_{X, S}(w) \neq 0$, we call $(P, w)$ a verter pair of $X$. It is well-known (see, for instance, Landrock [15, UI.3.2]) that the blocks of $k N_{G}(P)$ coincide with the primitive idempotents of $\left(k C_{G}(P)\right)^{N_{G}(P)}$. Furthermore, there is a bijective correspondence between the blocks $b$ of $k N_{G}(P)$ and the $N_{G}(P)$-orbits of blocks $w$ of $k C_{G}(P)$ whereby $b$ corresponds to the orbit of $w$ provided $b$ is the sum of the $N_{G}(P)$-conjugates of $w$. Now let $b$ be the block of $k N_{G}(P)$ fixing $Y$. Then $\theta_{Y, S}(b)=1$. In view of the way the Green correspondence preserves defect multiplicity modules, $\theta_{X, s}(b)=1$. So, given a block $w$ of $k C_{G}(P)$, the condition that $(P, w)$ is a vertex pair of $Y$, and the condition that $(P, w)$ is a vertex pair of $X$ are both equivalent to the condition that $b$ is the sum of the $N_{G}(P)$-conjugates of $w$. Since $G$ acts transitively on the vertices of $X$, we have recovered Sibley's observation $[28,2.5]$ that the vertex pairs of $X$ comprise a $G$-orbit of Brauer pairs on $k G$.

Let $(P, W)$ be a defect pair of $X$. Choosing the source $k P$-module $S$ such that $W$ occurs in $W_{G}(X, S)$, then $b$ fixes $W$ because $\theta_{X, S}(b)=1$. Letting $w$ be the block of $k C_{G}(P)$ fixing $W$, then $b w=w$, and $(P, w)$ must be a vertex pair of $X$. Comments above and Theorem 2.2 give: 
Lemma 23. Given an indecomposable $k G$-module $X$ with vertex pair $(P, w)$, defect pair $(P, W)$, and source $k P$-module $X$, then:

(a) The block $w$ of $k C_{G}(P)$ fixes some $N_{G}(P)$-conjugate of the simple $k C_{G}(P)$-module $W$.

(b) Let $Y$ be the indecomposable $k N_{G}(P)$-module with vertex $P$ in Green correspondence with $X$. Then $Y$ has vertex pair $(P, w)$, defect pair $(P, W)$, and source $k P$-module $S$.

(c) Let $b$ be the block of $k N_{G}(P)$ fixing $Y$. Then $b$ is the sum of the $N_{G}(P)$-conjugates of $w$. Also, $b$ fixes both $V_{G}(X, S)$ and $W$.

(d) Let $a$ be the block of $k G$ fixing $X$. Then $\operatorname{Br}_{P}^{G}(a) w=w$, and $\operatorname{Br}_{P}^{G}(a)$ fixes both $V_{G}(X, S)$ and $W$.

We say that $X$ is simply defective provided $V_{g}(X, S)$ is simple (and then all the defect multiplicity modules of $X$ are simple). Sufficient criteria for $X$ to be simply defective are, by Picaronny-Puig [20, Proposition 1], that $X$ is simple, or by $[2,1.2]$, that the vertices of $X$ coincide with the defect groups of the block of $k G$ fixing $X$. If $X$ is simply defective, and $W$ is a simple $k C_{G}(P)$-module, then $(P, W)$ is a defect pair of $X$ provided some $N_{G}(P)$-conjugate of $W$ occurs in the semisimple $k C_{G}(P)$-module

$$
W_{G}(X, S)=\operatorname{Res}_{C_{G}(P)}^{N_{G}(P, S)}\left(V_{G}(X, S)\right) \text {. }
$$

Lemma 2.4 (Picaronny-Puig). Let $X$ be a simply defective indecomposable $k G$-module.

(a) Each defect pair of $X$, and each vertex pair of $X$, is selfcentralizing.

(b) Let $P$ be a vertex of $X$. There is a bijective correspondence between the defect pairs of $X$ taking the form $(P, W)$, and the vertex pairs of $X$ taking the form $(P, w)$, whereby $(P, W) \leftrightarrow(P, w)$ provided $W$ is the isomorphically unique simple $k C_{G}(P)$-module.

Proof. Part (a) is shown in Picaronny-Puig [20, page 71]; see also Puig's clarification presented in the proof of $[2,1.1]$. Part (b) follows.

Given a $p$-group $Q$, we define a local $k Q$-module to be an indecomposable $k Q$-module with vertex $Q$. Given a local pair $(Q, W)$ on $k G$, and a local $k Q$-module $T$, then by Puig [24, 2.10.3, 9.12], there exists an indecomposable $k G$-module with defect pair $(Q, W)$ and source $k Q$ module $T$.

\section{Multiplicity modules and Fong's first correspondence}

Fixing a normal $p^{\prime}$-subgroup $R$ of $G$, and a block $e$ of $k R$ with stabilizer $T$ in $G$, then $e$ may be regarded as a central idempotent of $k T$, and the primitive idempotent $f:=\operatorname{Tr}_{T}^{G}(e)$ of $(k R)^{G}$ may be regarded as a central idempotent of $k G$. 
Cliff observed in [5, 1.6(iii)] that Fong's first correspondence provides an injective function from the isomorphism classes of indecomposable $k T e$-modules with vertex $P$, and the isomorphism classes of indecomposable $k G f$-modules with vertex $p$ (see Lemma 3.1). Also, this function is not surjective when $k T e$ has an indecomposable module with a vertex $G$-conjugate but not $T$-conjugate to $P$. Thus, when studying indecomposable $k G f$-modules with a given vertex, and seeking a reduction to $k T e$, there appears to be an irritating consideration of $G$-fusion in $T$. (In particular, this issue arises when counting simple $k G f$-modules with a given vertex.) Fortunately, confining attention to indecomposable $k G f$ modules with a given defect pair $(P, W)$, a felicitous reduction is provided by a bijective correspondence as follows. Assuming that $k G f$ has an indecomposable module with defect pair $(P, W)$, then by Lemma 2.3(d), $\operatorname{Br}_{P}^{G}(e)$ fixes $W$. Since $f$ is the sum of the $G$-conjugates of $e$, and $\mathrm{Br}_{P}^{G}$ annihilates $(k R)_{Q}^{P}$ for all $Q<P$, we have $\operatorname{Br}_{P}^{G}(f)=\sum_{e^{\prime}} \mathrm{Br}_{P}^{G}\left(e^{\prime}\right)$, where $e^{\prime}$ runs over the $P$-stable $G$-conjugates of $e$. So we can replace $e$ with a suitable $G$-conjugate so as to ensure that $\operatorname{Br}_{p}^{G}(e)$ does not annihilate $W$. Then (see Proposition 3.3), $k T$ has a defect pair of the form $\left(P, W_{T}\right)$ such that Fong's first correspondence restricts to a bijective correspondence between the isomorphism classes of indecomposable $k G f$-modules with defect pair $(P, W)$, and the isomorphism classes of indecomposable $k T e$-modules with defect pair $\left(P, W_{T}\right)$. Moreover, $W$ and $W_{T}$ are themselves related by a "local" case of Fong's first correspondence.

The induced algebra $\operatorname{Ind}_{T}^{G}(k T e)$ is, by Puig's definition [22, 3.3], the interior $G$-algebra $k G \underset{k T}{\bigotimes} k T e \bigotimes_{k T} k G$ with structural map given by $g \mapsto \sum_{g^{T \leq G}} g y \otimes 1 \otimes y^{-1}$ for $g \in G$, and with multiplication such that, for $x_{1}$, $y_{1}, x_{2}, y_{2} \in G$ and $\eta_{1}, \eta_{2} \in k T e$, the product $\left(x_{1} \otimes \eta_{1} \otimes y_{1}^{-1}\right)\left(x_{2} \otimes \eta_{2} \otimes\right.$ $\left.y_{2}^{-1}\right)$ vanishes unless $y_{1} T=x_{2} T$, in which case it equals $x_{1} \otimes$ $\eta_{1} y_{1}^{-1} x_{2} \eta_{2} \otimes y_{2}^{-1}$. Using the fact that ege $=0$ for all $g \in G-T$, it is easy to check that there is an interior $G$-algebra isomorphism

$$
\operatorname{Ind}_{T}^{G}(k T e) \cong k G f
$$

given by $x \otimes \eta \otimes y^{-1} \leftrightarrow x \eta y^{-1}$ for $x, y \in G$ and $\eta \in k T e$. We now see that the induction functor $\operatorname{Ind}_{T}^{G}$ on modules restricts to a Morita equivalence

$$
\operatorname{Ind}_{T}^{G}: \operatorname{Mod}(k T e) \rightarrow \operatorname{Mod}(k G f) .
$$

This is Fong's first correspondence; see Cliff [5, Section 1]. The inverse Morita equivalence is evidently given by the condition that, for any $k T e$-module $Y$ with corresponding $k G e$-module $X=\operatorname{Ind}_{T}^{G}(Y)$, we have $Y=e \operatorname{Res}_{T}^{G}(X)$. 
We shall also need a twisted group algebra version of Fong's first correspondence. Let $k_{\sharp} G$ be an $R$-normal twisted group algebra of $G$. Then the preimage $k_{\sharp} T$ of $T$ in $k_{\sharp} G$ is also $R$-normal. We can still regard $e, f$ as central idempotents of $k_{\sharp} T, k_{\sharp} G$, respectively, and the appropriate induction functor still restricts to a Morita equivalence

$$
\operatorname{Ind} G: \operatorname{Mod}\left(k_{\sharp} T e\right) \simeq \operatorname{Mod}\left(k_{\sharp} G f\right)
$$

with inverse Morita equivalence as described before.

We now show that the Glaubermann correspondence (the special case, as in Okuyama [19, page 311], pertaining to our scenario here) can be formulated in terms of the Brauer correspondence. (I have been unable to locate, in the existing literature, a clear discussion of this well-known observation.) Let us fix a $p$-subgroup $P$ of $T$ such that $k T e$ has an indecomposable module with vertex $P$, and define $c:=\operatorname{Br}_{P}^{G}(e)$. Then $c=\operatorname{Br}_{P}^{T}(e)$, so by Theorem 2.2, $c \neq 0$. Evidently, we may regard $c$ and $e$ as blocks of $k P C_{R}(P)$ and $k P R$, respectively, in Brauer correspondence with common defect group $P$. Hence, in fact, $c$ is a block of $k C_{R}(P)$. Furthermore, $N_{T}(P)$ is clearly the stabilizer of $c$ in $N_{G}(P)$. It is easy to check that the isomorphically unique simple $k C_{R}(P) c$-module is the Glaubermann correspondent of the isomorphically unique simple $k R e$ module (but we shall not use this fact).

LeMma 3.1. Given an indecomposable kTe-module $Y$ with vertex $P$ and source $k P$-module $S$, then the corresponding indecomposable $k G f$-module $X=\operatorname{Ind}_{T}^{G}(Y)$ has vertex $P$ and source $k P$-module $S$. Moreover, $Y$ is simply defective if and only if $X$ is simply defective.

Proof. Of course, $X$ has vertex $P$ and source $k P$-module $S$. Evidently, we may identify $k_{\sharp} N_{T}(P, S)$ with the preimage of $N_{T}(P, S)$ in $k_{\sharp} N_{G}(P, S)$ in such a way that $V_{T}(Y, S)$ is a direct factor of $\operatorname{Res}_{N_{T}(P, S)}^{\mathcal{N}_{G}(P, S)}\left(V_{G}(X, S)\right)$. The twisted group algebras $k_{\sharp} N_{T}(P, S)$ and $k_{\#} N_{G}(P, S)$ are $C_{R}(P)$-normal, and $N_{T}(P, S)$ is the stabilizer of $c$ in $N_{G}(P, S)$, so $c$ is a central idempotent of $k_{\sharp} N_{T}(P, S)$, and $d_{N}:=$ $\operatorname{Tr}_{N_{T}(P, S)}^{N_{G}(P, S)}$ is a central idempotent of $k_{\sharp} N_{G}(P, S)$. We have a case of Fong's first correspondence

$$
\operatorname{Ind}_{N_{T}(P, S):}^{N_{G}(P, S)} \operatorname{Mod}\left(k_{\sharp} N_{T}(P, S) c\right) \neg \operatorname{Mod}\left(k_{*} N_{G}(P, S) d_{N}\right) \text {. }
$$

By Lemma 2.3(c), $c$ fixes $V_{T}(Y, S)$, so

$$
\operatorname{Ind}_{N_{T}(P, S)}^{N_{O}(P, S)}\left(V_{T}(Y, S)\right) \cong V_{G}(X, S) \text {. }
$$

Now $C_{T}(P)$ is the stabilizer of $c$ in $C_{G}(P)$, and in particular, $c$ is a central idempotent of $k C_{T}(P)$. Let $d:=\operatorname{Tr}_{C_{T}}^{C_{C}(P)}(c)$, which is a central idempotent of $k C_{G}(P)$. Fong's first correspondence may be cast as

$$
\operatorname{Ind}_{C_{T}(P)}^{C_{G}(P)} \operatorname{Mod}\left(k C_{T}(P) c\right) \rightarrow \operatorname{Mod}\left(k C_{G}(P) d\right) .
$$


LEMMA 3.2. There is a bijective correspondence between the local pairs on $k G$ of the form $(P, W)$ with $W$ fixed by $d$, and the local pairs on $T$ of the form $\left(P, W_{T}\right)$ with $W_{T}$ fuxed by $c$, whereby $(P, W) \leftrightarrow\left(P, W_{T}\right)$ provided $W \cong \operatorname{Ind}_{C_{r}^{C(P)}}^{C_{C}(P)}\left(W_{T}\right)$, in which case, $(P, W)$ is self-centralizing if and only if $\left(P, W_{T}\right)$ is self-centralizing.

Proof. This is clear.

Proposition 3.3. Let $Y, X$ be indecomposable modules of $k T e, k G f$, respectively, which correspond as in Lemma 3.1. Given local pairs of the form $\left(P, W_{T}\right),(P, W)$ on $T, G$, respectively, which correspond as in Lemma 3.2, then $Y$ has defect pair $\left(P, W_{T}\right)$ if and only if $X$ has defect pair $(P, W)$.

Proof. Using the proof of Lemma 3.1, the uniqueness of defect multiplicity modules up to conjugacy, and a Frattini argument, it is not hard to show that $Y$ has vertex $P$ and source $k P$-module $S$ if and only if $X$ does. We may assume that these two equivalent conditions hold. Applying the inverse form of Fong's first correspondence, we get

$$
\operatorname{hd}\left(V_{T}(Y, S) \approx c \operatorname{Res}_{N_{T}(P, S)}^{N_{G}(P, S)}\left(\operatorname{hd}\left(V_{G}(X, S)\right)\right)\right. \text {. }
$$

Restricting to $C_{R}(P)$, and recognising Fong's first correspondence again,

$$
\operatorname{Ind}_{C_{R}(P)}^{C_{G}(P)}\left(W_{T}(Y, S)\right) \cong W_{G}(X, S) .
$$

\section{Multiplicity modules and Fong's second correspondence}

We continue to work with a normal $p^{\prime}$-subgroup $R$ of $G$, but now assume that the block $e$ of $k R$ is $G$-stable. Fong's second correspondence will be presented as a Morita equivalence of the form

$$
\operatorname{Mod}(k G \epsilon) \approx \operatorname{Mod}(k G e)
$$

where $G$ is a central extension of $G:=G / R$ by a finite cyclic $p^{\prime}$-group $E$, and $\epsilon$ is a block of $k E$. Let $P$ be a $p$-subgroup of $G$ such that $k G e$ has an indecomposable module with vertex $P$, and let $d:=\operatorname{Br}_{P}^{G}(e)$. (This is consistent with the notation in Section 3, indeed, in that notation, the $G$-stability of $e$ forces $c=d$ and $e=f$.) The discussion, in Section 3, of the Glaubermann correspondence shows that $d$ is an $N_{G}(P)$-stable block of $k C_{R}(P)$. Fong's second correspondence, with $N_{G}(P), C_{R}(P), d$ in place of $G, R, e$, respectively, is a Morita equivalence

$$
\operatorname{Mod}\left(k \underline{N_{G}(P)} \delta\right) \cong \operatorname{Mod}\left(k N_{G}(P) d\right)
$$

where $\widehat{N_{G}(P)}$ is a central extension of $N_{G}(P):=N_{G}(P) / C_{R}(P)$ by a finite

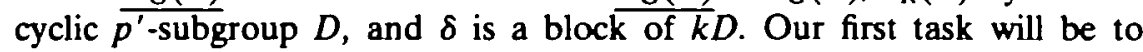
show how Puig's improvement of Dade's theorem, Theorem 4.1, allows 
us to choose $D=E, \delta=\epsilon$, and to choose $\widehat{N_{G}(P)}$ to coincide with the preimage of $N_{G}(P) R / R$ in $G$. Having set the machinery in this way, we shall find (see the proof of Proposition 4.8) that any simply defective indecomposable $k G e$-module $X$ with vertex $P$, and the corresponding indecomposable $k \hat{G} \epsilon$ module $\hat{X}$, have defect multiplicity modules which can be related by a formula involving an extension of a simple $k C_{R}(P) d$-module. Furthermore $\hat{X}$ is simply defective, has a vertex $\hat{P}$ canonically isomorphic to $P$, and the defect pairs of $X$ and of $\hat{X}$ are related in terms of Fong's second correspondence cast as a Morita equivalence

$$
\operatorname{Mod}\left(k C_{\zeta}(P) \epsilon\right) \approx \operatorname{Mod}\left(k C_{G}(P) d\right) .
$$

For any element $g \in G$, let $g$ denote the image of $g$ in $G$. The simple subalgebra $k R e$ of $k G e$ has centralizer $(k G e)^{R}$, so by Lemma 2.1 , there is an algebra isomorphism

$$
\bar{\phi}_{G, e}:(k G e)^{R} \otimes k R e \Im k G e
$$

given by $\mu \otimes \eta \mapsto \mu \eta$ for $\mu \in(k G e)^{R}$ and $\eta \in k R e$. Let us recall how $(k G e)^{R}$ acquires the structure of a twisted group algebra $k_{\sharp} G^{e}$ of $G$. Regarding $k G e$ as a $k(G \times G)$-module by left-right translation, the left translation action of $k G e$ on itself induces an algebra isomorphism $k G e \neg \operatorname{End}_{1 \times G}(k G e)$. By restriction, we obtain an algebra isomorphism $\rho:(k G e)^{R} \neg \operatorname{End}_{R \times G}(k G e)$ given by $\mu=(\rho(\mu))(e)$ for $\mu \in(k G e)^{R}$. Since $e$ is $G$-stable, the simple $k(R \times R)$-module $k R e$ is $G \times G$-stable. So $k G e=\bigoplus_{g^{R} \in G} g k R e$ as a direct sum of mutually isomorphic simple $k(R \times R)$-modules. In particular, each $(g k R e)^{R}$ is 1-dimensional. For each $g \in G$, we choose a non-zero element $\tilde{g}^{e} \in(g k R e)^{R}$. It is easy to check that $k_{*} G^{\boldsymbol{C}}:=(k G e)^{R}$ is a twisted group algebra of $G$ with proper basis $\bar{G}^{e}:=\left\{\tilde{s}^{e}: g \in G\right\}$.

Let $k_{\sharp} G^{e}$ be the inflation of $k_{\sharp} G^{e}$ to an $R$-normal twisted group algebra of $G$, and let $\bar{G}^{e}=\left\{\bar{g}^{e}: g \in G\right\}$ be the lift of $G^{e}$ to an $R$-normal basis of $k_{*} G^{e}$. This $k_{*} G^{e}$ is a $k_{*} G^{e}$-algebra via the epimorphism $k_{\sharp} G^{e} \rightarrow k_{\sharp} G^{e}$ given by $\tilde{g}^{e} \mapsto \tilde{g}^{e}$. We regard $k G e$ as an interior $G$-algebra via the projection $g \mapsto g e$. By Lemma 2.1, the $G$-algebra structure of $k R e$ provided by conjugation lifts to a $k_{\sharp} G^{\text {eo }}$-algebra structure in such a way that

$$
\phi_{G, e}: k_{\#} G^{e} \otimes k R e \rightrightarrows k G e
$$

is an interior $G$-algebra isomorphism via the canonical equivalence $k G \cong k_{\#} G^{e} * k_{\#} G^{\infty}$. The structural map $\theta_{G, e}: k_{\#} G^{\infty 0} \rightarrow K R e$ is determined by the condition that $g e=g^{e} \theta_{G, e}\left(\bar{g}^{e o}\right)$ for $g \in G$. Since $k_{\sharp} G^{e}$ is $R$-normal, so is $k_{\sharp} G^{\infty}$. For all $y \in \bar{R}$, we have $\tilde{y}^{e}=1$, hence $\theta_{G, e}(y)=y e$. That is to say, $\theta_{G, e}$ restricts to the structural map of the interior $R$-algebra $k$ Re. 
Given a subgroup $R \leqslant H \leqslant G$, we define $H:=H / R$ as a subgroup of $G$. Writing $k_{\sharp} \underline{H}^{e}, k_{\sharp} H^{e}, k_{*} H^{e 0}$ for the preimages of $\underline{H}, H, H$ in $k_{\sharp} \underline{G}^{e}$, $k_{\#} G^{e}, k_{\#} G^{e 0}$, respectively, then $k_{\#} \underline{H}^{*}=(k H e)^{R}$ is a $k_{\#} H^{e}$-algebra, $k R e$ is a $k_{\sharp} H^{e o}$-algebra, and $\tilde{\phi}_{G \text {.e }}$ restricts to the interior $H$-algebra isomorphism

$$
\tilde{\phi}_{H, e}: k_{\#} H^{r} \otimes k R e \Im k H e .
$$

In the parallel "local" situation, $k_{\sharp} N_{G}(P)^{d}:=\left(k N_{G}(P) d\right) R$ is a twisted group algebra of $N_{G}(P)$, we lift $k_{\sharp} \overline{N_{G}(P)^{d}}$ to a $C_{R}(P)$-normal twisted group algebra $k_{\sharp} \overline{N_{G}(P)^{d}}$ of $N_{G}(P)$, we realise $k C_{R}(P)$ as a $k_{\sharp} N_{G}(P)^{d o}$ algebra, and we have an interior $N_{G}(P)$-algebra isomorphism

$$
\Phi_{N_{G}(P), d}: k_{*} \underline{N_{G}(P)^{d}} \otimes k C_{R}(P) d \simeq k N_{G}(P) d .
$$

Given a subgroup $C_{R}(P) \leqslant L \leqslant N_{G}(P)$, we define $L:=L / C_{R}(P)$ as a subgroup of $N_{G}(P)$. (This is consistent with the notation above because if $R \leqslant L \leqslant N_{G}(\bar{P})$ then $C_{R}(P)=R$ and $L$ is defined unambiguously.) As before, $\tilde{\phi}_{N_{G}(P), d}$ restricts to an interior $L$-algebra isomorphism

$$
\Phi_{L, d}: k_{*} L^{d} \otimes k C_{R}(P) d \Im k L d .
$$

We define group epimorphisms $\pi^{d}: N_{G}(P) \rightarrow N_{G}(P)$ and $\pi^{e}: N_{G}(P) \rightarrow$ $N_{G}(P) R$, and define a group isomorphism $v: \underline{N_{G}(P)} \neg N_{G}(P) R$ where $\pi^{d}$ is the evident canonical epimorphism, $v$ is induced by the inclusion $N_{G}(P) \hookrightarrow N_{G}(P) R$, and $\pi^{e}:=v \pi^{d}$. Via $v$, we regard $k_{\sharp} N_{G}(P) R^{e}$ as a twisted group algebra of $N_{G}(P)$, and in particular, regard $\overline{k_{\sharp} C_{G}(P) R^{e}}$ as a twisted group algebra of $C_{G}(P)$.

In the following version of Dade's theorem, the construction of $\tilde{v}_{C}$ is implicit in Kulshammer-Robinson [14, page 101], while the extendibility of $\tilde{v}_{C}$ to an equivalence $\tilde{v}$ as specified is essentially Puig's improvement [23, (e)] of Dade [6, 10], [7, 9.1].

Theorem 4.1 (Dade, Puig). We have $k_{*} C_{G}(P) R^{e}=\left(k C_{G}(P) R e\right)^{P R}$. The Brauer map $\mathrm{Br}_{P}^{G}$ restricts to an equivalence

$$
\tilde{v}_{C}: k_{*} \underline{C_{G}(P) R^{e}} \neg k_{*} \underline{C_{G}(P)^{d}}
$$

of twisted group algebras of $C_{G}(P)$, Moreover, $\tilde{v}_{C}$ extends to an equivalence $\tilde{v}: k_{\sharp} \underline{N_{G}(P) R^{e}} \neg k_{\sharp} \underline{N_{G}(P)^{d}}$ of twisted group algebras of $\underline{N_{G}(P)}$.

Proof. The asserted equality holds because $P$ stabilises, and hence fixes, each of the 1-dimensional summands in

$$
k_{\sharp} \underline{C_{G}(P) R^{e}}=\bigotimes_{x C_{R}(P) \in C_{G}(P)}(x k R e)^{R} .
$$


Landrock [15, III.2.1], the identity $N_{P R}(P)=P C(R)$, and the fact that $P$ is a Sylow $p$-subgroup of both $P R$ and $P C_{R}(P)$, give $\operatorname{Br}_{P}^{G}\left(\left(k C_{G}(P) R\right)^{P R}\right)=\left(k C_{G}(P)\right)^{C_{n}(P)}$. So $\mathrm{Br}_{P}^{G}$ restricts to an algebra epimorphism $\tilde{v}_{C}$ with domain and codomain as specified. Clearly, $\tilde{v}_{C}$ is an equivalence.

Let $k_{\sharp} N_{G}(P)^{e}$ denote the preimage of $N_{G}(P)$ in $k_{\sharp} N_{G}(P) R^{e}$. Let $\mathcal{N}$ denote the normalizer in $(k R e)^{*}$ of the image of $P$ in $(k R e)^{*}$. Now $\mathrm{Br}_{P}^{G}$ restricts to a group epimorphism $\left((k R e)^{P}\right)^{*} \rightarrow\left(k C_{R}(P) d\right)^{*}$ which, by [23, (e)], extends to a group epimorphism $\gamma: \mathcal{N} \rightarrow\left(k C_{R}(P) d\right)^{*}$ such that $\gamma(t) \operatorname{Br}_{P}^{G}(\gamma) \eta\left(t^{-1}\right)=\operatorname{Br}_{P}^{G}\left(t \eta t^{-1}\right)$ for all $t \in \mathcal{N}$ and $\eta \in(k R e)^{P}$. By the uniqueness property of pull-backs, there exists a unique equivalence

$$
\tilde{\mu}^{o}: k_{\#} N_{G}(P)^{\infty} \cong k_{\sharp} N_{G}(P)^{d o}
$$

such that $\theta_{N_{G}(P) . d}\left(\tilde{\mu}^{o}\left(\tilde{g}^{e o}\right)\right)=\gamma\left(\theta_{G, e}\left(\tilde{g}^{e o}\right)\right)$ for all $g \in N_{G}(P)$. It is easily checked that $\tilde{\mu}^{\circ}$ is $C_{R}(P)$-normal. Therefore, we can choose the proper basis $N_{G}(P)^{d}$ of $k_{\#} N_{G}(P)^{d}$ in such a way that $\tilde{\mu}^{o}\left(\tilde{g}^{e o}\right)=\tilde{g}^{d o}$ for all $g \in N_{G}(P)$. The opposite equivalence of $\tilde{\mu}^{o}$ is the $R$-normal equivalence $\tilde{\mu}: k_{\sharp} N_{G}(P)^{e} \Im k_{\sharp} N_{G}(P)^{d}$ given by $\tilde{\mu}\left(\tilde{g}^{e}\right)=\tilde{g}^{d}$. Straightforward manipulations confirm that $\tilde{\mu}$ induces, via $\pi^{e}$ and $\pi^{d}$, an equivalence $\tilde{v}$ with the required properties.

Theorem 1.2 will be proved by an inductive argument making use of the fact that $p$-soluble groups are closed under subgroups, factor groups, and extension. To prepare for this, the twisted group algebras in Dade's theorem will be replaced with central extensions by a cyclic $p^{\prime}$-group. Let us choose, and fix, a finite cyclic $p^{\prime}$-group $E$ of order divisible by $|\underline{G}|_{p^{\prime}}$. Let $\epsilon$ be any block of $k E$ such that the $k E \epsilon$-modules are faithful, so that $E$ acts on any $k E \epsilon$-module via a group monomorphism $E \rightarrow k^{*}$. It is well-known that the exponent of the abelian group $H^{2}\left(G, k^{*}\right)$ divides $|G|_{p^{\prime}}$, so we can choose the respective proper bases $G^{e}, \tilde{G}^{e}, \bar{G}^{e o}$ of $k_{\sharp} G^{e}$, $k_{\sharp} G^{e}, k_{\sharp} G^{e o}$ such that the factor set $\alpha^{e}: G \times G \rightarrow k^{\star}$ associated with $G^{e}$ takes values in the image of $E$. Then the $R$-normal bases $k_{\#} G^{e}, k_{*} G^{e o}$ of the $R$-normal twisted group algebras $k_{\sharp} G^{e}, k_{\sharp} G^{e o}$ have factor sets of $\alpha^{e}$, $\alpha^{e o}$, respectively, satisfying $\underline{\alpha}^{e}(g, h)=\alpha^{e}(g, h)=\alpha^{e o}(g, h)^{-1}$ for all $g$, $h \in G$. Let $1 \rightarrow E \rightarrow G \rightarrow G \rightarrow 1$ be a central extension of $G$ by $E$ such that $G$ has a section $\{g: g \in \underline{G}\}$ in $G$ with the property that each $\hat{g} \hat{g} \widehat{g h}^{-1}$ is the element of $E$ with image $\alpha^{e}(g, h)$ in $k^{*}$. We have an algebra isomorphism $\psi_{G, e}: k \underline{G} \in \neg k_{\sharp} G^{e}$ given by $\bar{g} \epsilon \mapsto \bar{g}^{e}$ for all $g \in G$. We impose a $k_{\#} G^{e}$-algebra structure on $k G \epsilon$ by insisting that $\psi_{G, e}$ is a $k_{\sharp} G^{e}$-algebra isomorphism. The $k_{\sharp} G^{e}$-algebra structure of $k G \epsilon$, and the interior $\hat{G}$-algebra structure of $k \hat{G} \epsilon$ clearly induce the same $G$-algebra structure on $k \hat{G} \epsilon$. Let $\hat{\phi}_{G, e}:=\bar{\phi}_{G, e}\left(\psi_{G, e} \otimes 1\right)$, which is an interior $G$-algebra 
isomorphism $\hat{\phi}_{G, e}: k G \varepsilon \otimes k R e \Im k G e$. Since $k R e$ is a simple algebra, $\hat{\phi}_{G, e}$ provides a Morita equivalence

$$
\Phi_{G, e}: \operatorname{Mod}(k \underline{G} e) \simeq \operatorname{Mod}(k G e) \text {. }
$$

This is Fong's second correspondence; see Cliff [5, Section 2].

Given a subgroup $R \leqslant H \leqslant G$, we let $\hat{\theta}$ denote the preimage of $H=H / R$ in $\underline{G}$. Note that $\tilde{\phi}_{H, e}, \psi_{H, e}, \hat{\phi}_{H, e}$ are restrictions of $\tilde{\phi}_{G, e} \psi_{G, e}$ $\overline{\hat{\phi}}_{G, e}$, respectively. Given a $k \hat{\underline{\theta}} \epsilon$-module $\underline{\underline{Y}}$, we write $\underline{\underline{Y}}$ e to denote $\underline{\underline{Y}}$ regarded as a $k_{\sharp} H^{e}$-module via $\psi_{H, e}$ and inflation, that is via the composite map $k_{\sharp} H^{e} \rightarrow k_{\sharp} \underline{H}^{e} \rightarrow k \hat{H} \varepsilon$. Let $\mathscr{S}_{R}^{e}$ be a simple $k R e$-module, and let $\mathscr{S}_{H}^{e}$ denote $\mathscr{S}_{R}^{e}$ regarded as a $k_{t H} H^{e 0}$-module via the structural map $\theta_{H, e}$ (This notation makes sense because $k_{\sharp} H^{e o}$ is $R$-normal, and $\theta_{R, e}$ is the structural map of $k R e$ as an interior $R$-algebra.) We have $\Phi_{H, e}(\underline{Y})=$

$\underline{Y}^{e} \otimes \mathscr{S}_{H}^{e}$. We can now see that $\Phi_{H, e}, \Phi_{G, e}$ commute with the restriction functors

$$
\operatorname{Res}_{H}^{G}: \operatorname{Mod}(k G e) \rightarrow \operatorname{Mod}(k H e), \quad \operatorname{Res}_{G}^{\xi}: \operatorname{Mod}(k \underline{G} \epsilon) \rightarrow \operatorname{Mod}(k \underline{H} \epsilon),
$$
and that $\Phi_{H, e}, \Phi_{G, e}$ commute with the induction functors

$$
\operatorname{Ind}_{H}^{G}: \operatorname{Mod}(k H e) \rightarrow \operatorname{Mod}(k G e), \quad \operatorname{Ind} \hat{\theta}^{\delta}: \operatorname{Mod}(k \underline{H}) \rightarrow \operatorname{Mod}(k \underline{G} \epsilon) .
$$

Working with $N_{G}(P)$ in place of $G$, we choose a finite cyclic $p^{\prime}$-group $D$ of order divisible by $\left|N_{G}(P)\right|_{p^{\prime}}$, and choose a block $\delta$ of $k D$ such that the $k D \delta$-modules are faithful. As before, we obtain a central extension $1 \rightarrow D \rightarrow \widehat{N_{G}(P)} \rightarrow N_{G}(P) \rightarrow 1 \quad$ of $\quad N_{G}(P)=N_{G}(P) / C_{R}(P)$ by $D, \quad$ a $k_{\sharp} N_{G}(P)^{d}$-algebra isomorphism $\psi_{N_{G}(P), d}: k N_{G} \widehat{(P)} \delta \Im k_{\sharp} N_{G}(P)^{d}$, an in-

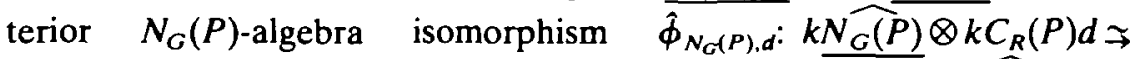
$k N_{G}(P) d$, and a Morita equivalence $\Phi_{N_{G}(P), d}: \overline{\operatorname{Mod}}\left(k N_{G(P)} \delta\right) \neg$ $\operatorname{Mod}\left(k N_{G}(P) d\right)$.

We now harness the power of Dade's equivalence. As in the proof of Theorem 4.1, we may insist that the proper basis $G^{d}$ of $k_{*} G^{d}$ satisfies $v\left(g^{e}\right)=\pi^{d}(g)^{d}$ for all $g \in N_{G}(P)$. Then the factor set $\underline{\alpha}^{d}: N_{G}(P) \times$

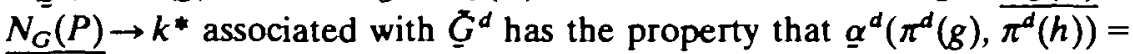
$\underline{\alpha}^{e}(\underline{g}, \underline{h})$ for all $g, h \in N_{G}(P)$. We are free to choose any finite cyclic $P^{\prime}$-group $D$ and block $\delta$ of $k D$ satisfying the stipulations above, and

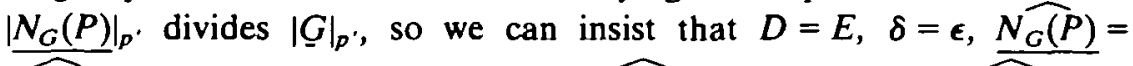
$\widehat{N_{G}(P) R}$, that the epimorphisms $\underline{N_{G}(P)} \rightarrow \underline{N_{G}(P)}$ and $\underline{N_{G}(P) R} \rightarrow$ $\overline{N_{G}(P) R}$

commute with $v$, and that $\psi_{N_{G}(P), d}$ and $\psi_{N_{G}(P), c}$ commute with $\tilde{v}$.

Given a subgroup $C_{R}(P) \leqslant L \leqslant N_{G}(P)$, writing $\hat{L}$ for the preimage of $\underline{L}=L / C_{R}(P)$ in $\widehat{N_{G}(P)}=\widehat{N_{G}(P) R}$, then $\underline{L}=\widehat{L R}$. The $k_{*} L^{d}$-algebra isomorphism $\psi_{L, d}: k \underline{k} \neg \neg k_{\sharp} L^{d}$, commutes with $\psi_{L R, e}$ and $\bar{v}$. For any $k \underline{L} \epsilon$-module $\underline{\hat{Y}}$, we write $\underline{\underline{Y}}^{d}$ to denote $\underline{\hat{Y}}$ regarded as a $k_{*} \underline{L}^{d}$-module 
via $\psi_{L, d}$ and inflation. The Morita equivalence $\Phi_{L, d}: \operatorname{Mod}(k \underline{\underline{L} \varepsilon) \rightrightarrows}$ $\operatorname{Mod}(k L d)$ is given by $\Phi_{L, d}(\underline{Y})=\underline{P}^{d} \otimes \mathscr{S}_{L}^{d}$.

We have $\widehat{P R}=\hat{P} E$, where $\hat{P}:=O_{P}(\widehat{P R})$. The epimorphisms $G \rightarrow G$ and $G \rightarrow G$ restrict to isomorphisms $P \approx P R \equiv \hat{P}$. Let $P R \leqslant H \leqslant G$, and let $Y, \underline{\underline{Y}}$, be modules of $k H e, k \hat{H} \epsilon$, respectively, which correspond via the Morita equivalence $\Phi_{H . e}$ Since $\Phi_{P R, e}$ and $\Phi_{H, e}$ commute with induction and restriction, $Y$ is $P R$-projective if and only if $\underline{Y}$ is $\underline{P R}$-projective. But $P, \hat{P}$ are Sylow $p$-subgroups of $P R, \widehat{P R}$, respectively, so $Y$ is $P$-projective if and only if $\underline{\underline{Y}}$ is $P$-projective. This conclusion still holds when $P$ is replaced with any subgroup of $P$, so we recover the following version of Cliff [5, 2.5(iii)].

Lemma 4.2 (Cliff). For $P R \leqslant H \leqslant G$, the Morita equivalence $\Phi_{H, e}$ restricts to a bijective correspondence between the isomorphism classes of indecomposable $k H e$-modules with vertex $P$, and the isomorphism classes of indecomposable $k \hat{H} \epsilon$-modules with vertex $\hat{P}$.

Given a local $k P$-module $S$, then since $N_{P R}(P)=P C_{R}(P)$, we can form the indecomposable $k N_{P R}(P) d$-module $S \otimes \mathscr{S}_{C_{R}(P)}$, which has vertex $P$. We write $S_{P R}$ to denote the indecomposable $k P R$-module in Green correspondence with $S \otimes \mathscr{P}_{C_{R}(P)}^{d}$

LEMMA 4.3. There is a bijective correspondence between the isomorphism classes of local $k P$-modules $S$, and the isomorphism classes of indecomposable $k P R e$-modules $T$ with vertex $P$, whereby $S \leftrightarrow T$ provided $T \cong S_{P R}$.

Proof. This is easy to prove by considering the evident algebra isomorphism $k N_{P R}(P) d \cong k P \otimes k C_{R}(P) d$.

For any local $k P$-module $S$, we write $S$ to denote a local $k \hat{P}$-module such that $\Phi_{P R, e}\left(S_{\overrightarrow{P R}}\right) \approx S_{P R}$.

LEMMA 4.4. There is a bijection correspondence between the isomorphism classes of local $k P$-modules and the isomorphism classes of local $k \hat{P}$-modules, whereby each local $k P$-module $S$ corresponds to $S$.

Proof. This is immediate from Lemmas 4.2 and 4.3.

Lemma 4.5. $N_{G}(P, S)=\underline{N_{G}(P, S)}$ for any local $k P$-module $S$.

Proof. This follows quickly from the fact that $\Phi_{P R, e}$ respects the conjugation actions of $N_{G}(\underline{P R})$ on $\operatorname{Mod}(\widehat{\underline{P R} \epsilon})$ and on $\operatorname{Mod}(k P R e)$.

LeMMA 4.6. Let $S$ be a local $k P$-module. The isomorphism classes of indecomposable $k G e$-modules with vertex $P$ and source $k P$-module $S$ are, 
via $\Phi_{G, e}$ in bijective correspondence with the isomorphism classes of indecomposable $k G \epsilon$-modules with vertex $\hat{P}$ and source $k \hat{P}$-module $S$.

Proof. Let $\hat{X}$ be an indecomposable $k \underline{G} \epsilon$-module, and let $X=$ $\Phi_{G . e}(\underline{X})$. Lemma 4.2 says that $X$ has vertex $P$ if and only if $\underline{X}$ has vertex $\hat{P}$. Suppose now that this condition holds. It is easy to show that $X$ has source $k P$-module $S$ if and only if $S_{P R}$ is a direct factor of $\operatorname{Res}_{P R}^{G}(X)$. Similarly, $\underline{X}$ has source $k \hat{P}$-module $S$ if and only if $\hat{S}_{\widehat{P Q}}$ is a direct factor of $\operatorname{Res}_{\bar{p} \bar{R}}^{\delta}(\underline{X})$. The assertion follows because $\Phi_{P R, e}$ and $\bar{\Phi}_{G, e}$ commute with restriction.

We have $C_{\zeta}(\hat{P})=\widehat{C_{G}(P)}$, so

$$
\Phi_{C_{G}(P), d}: \operatorname{Mod}\left(k C_{\zeta}(\beta) \epsilon\right) \simeq \operatorname{Mod}\left(k C_{G}(P) d\right)
$$

is a Morita equivalence. Let $\underline{W}$ be a simple $k C_{\phi}(\hat{P}) \epsilon$-module, and let $W=\Phi_{C_{G}(P), d}(W)$ be the corresponding simple $k C_{G}(P) d$-module. Observing that $Z(\hat{P})=O_{P}\left(Z(P) \hat{C}_{R}(P)\right)$, Lemma 4.2 shows that $\hat{W}$ has vertex $Z(\hat{P})$ if and only if $W$ has vertex $Z(P)$. The following result is now clear.

Lemma 4.7. There is a bijective correspondence between the selfcentralizing local pairs on $k G$ of the form $(P, W)$ with $W$ fixed by $d$, and the self-centralizing local pairs on $k \hat{G}$ of the form $(\hat{P}, \hat{W})$ with $W$ fixed by $\epsilon$, whereby $(P, W) \leftrightarrow(\hat{P}, \underline{W})$ provided the simple modules $W, W$ of $k C_{G}(P) d, k C_{G}(\hat{P}) \epsilon$, respectively, correspond via $\Phi_{C_{G}(P), \boldsymbol{d}}$

PRoposition 4.8. Let $X$ be a simply defective indecomposable $k G e$ module with defect pair $(P, W)$, let $\underline{X}:=\Phi_{G, e}^{-1}(X)$, and let $(P, W)$ be the self-centralizing local pair corresponding to $(P, W)$, as in Lemma 4.7. Then $\underline{X}$ is simply defective with defect pair $(\hat{P}, \underline{W})$.

Proof. We sketch the argument. By Lemma 2.3(d), $d$ fixes $W$. By Lemma $2.4(\mathrm{a}),(P, W)$ is self-centralizing, so the definition of $(\mathcal{P}, W)$ makes sense. Let $S$ be a source $k P$-module of $X$ such that $W$ occurs in the semisimple $k C_{G}(P)$-module $W_{G}(X, S)$. Lemma 4.6 tells us that $\hat{X}$ has vertex $\hat{P}$ and source $k \hat{P}$-module $\hat{S}$.

Via the equality $\underline{X}^{e} \otimes \mathscr{S}_{G}^{e}=X$, and evident isomorphisms $\operatorname{End}_{k}\left(\hat{X}^{e}\right) \approx$ $\operatorname{End}_{k}(\hat{X})$ of $k_{\sharp} G^{e}$-algebras, and $\operatorname{End}_{k}\left(\mathscr{S}_{G}^{e}\right) \cong k R e$ of $k_{\sharp} G^{e \infty}$-algebras, we obtain an interior $G$-algebra isomorphism $\tau$ : $\operatorname{End}_{k}(\underline{X}) \otimes k R e \Im$ $\operatorname{End}_{k}(X)$. Following through the proof of $[3,8.3]$, and using the simplicity of $V_{G}(X, S)$, we obtain an $N_{G}(P, S)$-algebra isomorphism

$$
\sigma: \Pi_{\mathcal{C}}(\underline{\hat{X}}, \hat{S}) \otimes k C_{R}(P) d \Im \Pi_{G}(X, S)
$$

given by $\sigma\left(s \&, s(\zeta) \otimes \mathrm{Br}_{P}^{G}(\eta)\right)=s_{X, S}(\tau(\zeta \otimes \eta))$ for $\zeta \in \operatorname{End}_{k \beta}(\underline{\underline{X}})$ and $\eta \epsilon$ $(k R e)^{P}$. The $N_{G}(P, S)$-algebra structure of the simple algebra $\Pi_{\zeta}(\underline{X}, \hat{S})$ lifts to a $k_{*} N_{G}(P, S)^{\prime}$-algebra structure for some twisted group algebra 
$k_{*} N_{G}(P, S)^{\prime}$ of $N_{G}(P, S)$. The uniqueness property of pull-backs provides an equivalence

$$
\tilde{\sigma}: k_{\sharp} N_{G}(P, S)^{\prime} * k_{\sharp} N_{G}(P, S)^{d o} \neg k_{\sharp} N_{G}(P, S)
$$

such that $\sigma$ and $\tilde{\sigma}$ commute with the two structural maps

$$
\begin{gathered}
k_{*} N_{G}(P, S)^{\prime} * k_{\sharp} N_{G}(P, S)^{d o} \rightarrow \Pi_{G}(\hat{X}, S) \otimes k C_{R}(P) d, \\
k_{*} N_{G}(P, S) \rightarrow \Pi_{G}(X, S) .
\end{gathered}
$$

Therefore $\underline{X}$ is simply defective.

In the notation of the proof of Theorem 4.1, we regard the domain of $\sigma$ as an interior $C_{G}(P)$-algebra by regarding $\Pi_{\hat{Q}}(\underline{X}, \hat{S})$ as a $k_{\#} \underline{C_{G}(P)^{d}}$ algebra via $\tilde{\mu}$. We have

$$
\theta_{N_{G}(P), d}\left(\tilde{\mu}^{o}\left(\tilde{x}^{e o}\right)\right)=\gamma\left(\theta_{G, e}\left(\tilde{x}^{e o}\right)\right)=\operatorname{Br}_{P}^{G}\left(\theta_{G, e}\left(\tilde{x}^{e o}\right)\right)
$$

for all $x \in C_{G}(P)$. So $\sigma$ is an interior $C_{G}(P)$-algebra isomorphism. Identifying $\Pi_{\zeta}(\underline{X}, S), \quad \Pi_{G}(X, S), \quad k C_{R}(P) d$ with $\operatorname{End}_{k}\left(W_{\zeta}(\underline{X}, S)\right.$ ), $\operatorname{End}_{k}\left(W_{G}(X, S)\right), \operatorname{End}_{k}\left(\mathscr{P}_{C_{\sigma}(P)}^{d}\right)$, respectively, then $\sigma$ determines a $k C_{G}(P)$-isomorphism

$$
\Phi_{C_{G}(P), d}\left(W_{G}(\underline{X}, S)\right) \approx W_{G}(X, S) .
$$

Therefore $\underline{W}$ occurs in $W_{\hat{G}}(\underline{X}, \hat{S})$, and $\underline{X}$ has defect pair $(\hat{P}, \underline{W})$.

I do not know whether the image under $\Phi_{G, e}$ of a simply defective indecomposable $k G \in$-module need be simply defective. (Note that the construction of $\sigma$ in the argument above relies on $X$ being simply defective.)

COROLlary 4.9. Let $(P, W)$ be a self-centralizing local pair on $k G$ such that there exists an indecomposable $k G e-m o d u l e$ with vertex $P$. Let $(\hat{P}, \hat{W})$ be the self-centralizing local pair on $k G$ corresponding to $(P, W)$ as in Lemma 4.7. Then $\Phi_{G, e}$ provides a bijective correspondence between the isomorphism classes of simple $k G$-modules with defect pair $(P, W)$, and the isomorphism classes of simple $k \hat{G}$-modules with defect pair $(\hat{P}, \underline{W})$.

Proof. By Lemma 2.3(d), e fixes every simple $k G$-module with defect pair $(P, W)$. Similarly, $\epsilon$ fixes every simple $k G \epsilon$-module with defect pair $(\hat{P}, \underline{W})$. The assertion is now an immediate consequence of Proposition 4.8.

\section{Proof of the main result}

Lemmas 2.3(c) and 2.4(a) tell us that, for any simple $k G$-module $X$ with vertex $P$, the vertex pairs of $X$ are self-centralizing, and uniquely determine the block of $k N_{G}(P)$ fixing the Green correspondent of $X$. So Theorem 1.1 is equivalent to Theorem 1.2. By Theorem 2.2, Theorem 
1.1 implies Corollary 1.3(a) which, in turn, clearly implies Corollary 1.3(b). Theorems 1.1 and 2.2 also give the equivalence of conditions (1) and (2) of Corollary 1.4. To complete the proof that Theorem 1.1 implies Corollary 1.4, we put $p=2$, and give an example of a finite soluble group $G$ with a 2-subgroup $P$ satisfying condition (1) of Corollary 1.4. This is a slight simplification of an example communicated to me by Isaacs so, while the ideas in the construction are due to him, any mistakes are my responsibility. (Isaacs has also communicated to me another example based on a group of order $2^{7} 3^{3}$.)

Let $A$ be a copy of $A_{4}$, let $V$ be the Klein subgroup of $A$, and let $K$ be the $A$-module of order 27 obtained by induction from a non-trivial $V$-module of order 3 . Then $K$ is a direct product of three groups $U, U^{\prime}$, $U^{n}$ of order 3 such that, on each of $U, U^{\prime}, U^{\prime \prime}$, exactly two of the involutions in $V$ acts non-trivially. Let $Q$ be the group generated by the involution fixing $U$, and define $Q^{\prime}$ similarly for $U^{\prime}$, so that $V=Q \times Q^{\prime}$. Let $H$ be the semidirect product $K A$. We have $N_{H}(Q)=U Q^{\prime} \times Q$ and $U Q^{\prime} \cong S_{3}$. Let $H$ act faithfully on a finite 2-group $F$. Let $A / V$ act non-trivially on a group $E$ of order 7 , and inflate this to an action of $H$. We construct the semidirect product $G:=(E \times F) H$, and let $P:=F Q$.

Now $N_{G}(P)=(E \times F) N_{H}(Q)=(E \times F) U V$, so $E$ is a central Sylow 7-subgroup of $N_{G}(P)$, and $N_{G}(P)$ is the direct product of $E$ and the unique Hall 7'-subgroup $L:=F N_{H}(Q)$. We have $L / F \cong N_{H}(Q) \cong S_{3} \times Q$, so $k L$ has a unique block, hence $k N_{G}(P)$ has precisely seven blocks, all with Sylow defect group $R:=F V$, and the blocks $b_{1}, b_{2}, b_{3}, b_{4}, b_{3}, b_{6}, b_{7}$ of $k N_{G}(P)$ coincide with the blocks of $k E$. Moreover, $k L$ has a simple module $Y$ with vertex $P$. For $1 \leqslant i \leqslant 7$, tensoring a simple $k E b_{i}$-module with $Y$, we obtain a simple $k N_{G}(P) b_{i}$-module $Y_{i}$ with vertex $P$. Using Theorem 2.2, it can be shown that each $Y_{i}$ lies in a block of $k G$ with defect group $R$. So it suffices to show that $k N_{G}(R)$ has less than seven blocks. Now $N_{G}(R)=(E \times F) A$, and the quotient $\bar{N}_{G}(R)$, being nonabelian of order 21 , has precisely four conjugacy classes. Perforce, $k N_{G}(R)$ has at most four blocks.

Having now shown that Theorem 1.2 implies the other three results stated in Section 1, we end with a three-step proof of Theorem 1.2. Suppose that $G$ is $p$-soluble, and let $(P, W)$ be a self-centralizing local pair on $k G$. Writing $l_{G}(P, W)$ for the number of isomorphism classes of simple $k G$-modules with defect pair $(P, W)$, Theorem 1.2 is, by Lemma 2.4(b), the assertion that

$$
l_{G}(P, W)=l_{N_{G}(P)}(P, W) .
$$

We argue by a triple induction, firstly on $\left.\mid G: O_{p}(G)\right)_{p}$, secondly on $|G|_{p}$, thirdly on $|G|$. If $O_{p}(G) \neq 1$, let $R:=O_{p}(G)$, otherwise let $R:=O_{p} \cdot(G)$. For any $R \leqslant H \leqslant G$, let $\underline{H}:=H / R$. 
First, let us suppose that $1<R=O_{p}(G)$. We may then assume that $P \leqslant R$, because otherwise $l_{G}(P, W)=0=l_{N_{G}(P)}(P, W)$. Let $N_{G}(P) \leqslant H \leqslant$ $G$, and let $X$ be a simple $k H$-module with vertex $P$ and source $k P$-module $S$. We have inflations $X=\inf _{H}^{H}(\underline{X})$ and $S=\inf _{P}^{P}(\underline{S})$ for a simple $k \underline{H}$-module $X$ with vertex $\underline{P}$ and source $k \underline{P}$-module $\underline{S}$. Identifying $\bar{N}_{C}(\underline{P})$ with $\bar{N}_{G}(P)$, then $\bar{C}_{G}(P) \leqslant \bar{C}_{G}(P)$. It is not hard to show that

$$
\left.\operatorname{Res}_{\bar{C}_{G}(P)}^{\overline{\bar{N}}_{H}(P, S)}\left(\bar{V}_{H}(X, S)\right)=\operatorname{Res}_{\bar{N}_{\sigma}}^{\bar{N}_{G}(P, S)} \bar{V}_{H}(X, \underline{S})\right) \text {. }
$$

This may be rewritten as

$$
W_{H}(X, S)=\operatorname{Res}_{C_{\sigma}(P)}^{C_{G}(P) R}\left(\inf _{\underline{C_{G}^{C}(P) R}}^{C_{C}(P)}\left(\operatorname{Res}_{\underline{C_{G}}}^{C_{C}(P)_{R}}\left(W_{H}(X, \underline{S})\right)\right)\right) \text {. }
$$

By Clifford's theorem, $l_{H}(P, W)=\sum_{W} l_{H}(\underline{P}, \underline{W})$, where $W$ runs over representatives of the $N_{G}(P)$-orbits of the isomorphism classes of simple $k C_{G}(\underline{P})$-modules with vertex $Z(\underline{P})$ such that

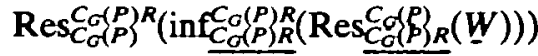

is a sum of $N_{G}(P)$-conjugates of $W$. Induction gives $l_{G}(P, W)=$ $l_{N_{G}(P)}(P, W)$ in this case.

We may suppose, henceforth, that $1=O_{p}(G)<R=O_{p},(G)<G$. Let $f$ be the primitive idempotent of $(k R)^{G}$ such that the central idempotent $\operatorname{Br}_{P}^{G}(f)$ of $k C_{G}(P)$ fixes $W$. Since $\operatorname{Br}_{P}^{G}(f)=\sum_{c} \operatorname{Br}_{P}^{G}(e)$, where $e$ runs over the $P$-stable blocks of $k R$ such that $f e=e$, we can choose a block $e$ of $k R$ with stabilizer $T$ in $G$ such that $P \leqslant T, f=\operatorname{Tr}_{T}^{G}(e)$, and $\operatorname{Br}_{P}^{G}(e)$ does not annihilate $W$. Let $c:=\operatorname{Br}_{P}^{G}(e)$ and $d:=\operatorname{Tr}_{C_{T}}^{C_{C}(P)}(c)$ as in Section 3. Then the block $d$ of $k C_{G}(P)$ fixes $W$. Let $W_{T}:=c \operatorname{Res}_{C_{T}(P)}^{C_{G}(P)}(W)$, which is the simple $k C_{T}(P)$-module such that $\left(P, W_{T}\right)$ is the self-centralizing local pair on $k T$ corresponding to $(P, W)$ as in Lemma 3.2. By Lemma 2.3(d), $f$ fixes all the simple $k G$-modules with defect pair $(P, W)$. Similarly, $e$ fixes all the simple $k T$-modules with defect pair $\left(P, W_{T}\right)$. If $T \neq G$, then Proposition 3.3 and induction give

$$
l_{G}(P, W)=l_{T}\left(P, W_{T}\right)=l_{N_{T}(P)}\left(P, W_{T}\right) l_{N_{G}(P)}(P, W)=l_{N_{T}(P)}\left(P, W_{T}\right) .
$$

On the other hand, if $T=G$, then by the identity $N_{C}(\beta)=\widehat{N_{G}(P)}$, the inductive hypothesis, and Corollary 4.9,

$$
l_{G}(P, W)=l_{G}(P, \underline{W})=l_{\underline{N_{G}(P)}}(\mathcal{P}, \underline{W})=l_{N_{G}(P)}(P, W) .
$$

\section{Acknowledgements}

The idea of refining the $p$-soluble case of Alperin's conjecture by considering multiplicity modules of simple modules originated with Puig. Much of the strategy behind this work arose during long conversations with him in 1992 and 1993. Letters from him and Isaacs in 1995 and 1996 
contained several valuable suggestions. I also thank the referee for some corrections and useful comments.

\section{REFERENCES}

1. J. L Alperin, 'Weights for finite groups', Proc. Symp. Pure Math. 17 (1987), 369-379.

2 L. Barker, 'Modules with simple multiplicity modules', J. Algebra 172 (1995), 152-158.

3. L. Barker, ' $G$-algebras, Clifford theory, and the Green correspondence', J. Algebra 172 (1995) 335-353.

4. L. Barker, 'Some remarks on defect pairs', (in preparation).

5. G. H. Cliff, 'On modular representations of $p$-solvable groups', J. Algebra 47 (1977), 129-137.

6. E. C. Dade, 'A correspondence of characters', Proc. Symp. Pure Math 37 (1980), $401-403$.

7. E. C. Dade, 'Extending endo-permutation modules', (typescript 1983).

8. E. C. Dade, 'Counting characters in blocks, l', Inventiones Math. 109 (1992), 187-210.

9. P. G. Gres', 'Fong's reduction, the correspondences of Brauer and Glaubermann and Alperin's weight conjecture', Contemporary Math. 131 (1992), 137-158.

10. I. M. Isaacs and G. Navarro, 'Weights and vertices for characters of $\pi$-separable groups', J. Algebra 177 (1995), 339-366.

11. R. Knorr, 'On the vertices of irreducible modules', Annals Math. 110 (1979), 487-499.

12. R. Knorr and G. R. Robinson, 'Some remarks on a conjecture of Alperin', J. London Math. Soc. (2) 39 (1989), 48-60.

13. B. Kulshammer and L. Puig, 'Extensions of nilpotent blocks', Inventiones Math. 102 (1990), 17-71.

14. B. Kulshammer and G. R. Robinson, 'On blocks of finite groups with a certain factorisation', Archiv. Math. 46 (1986), 97-101.

15. P. Landrock, Finite group algebras and their modules, London Math. Soc. Lecture Notes Series 84, Cambridge University Press (1983).

16. O. Manz and T. R. Wolf, Representations of soluable groups, London Math. Soc. Lecture Notes Series 185, Cambridge University Press (1993).

17. G. Navarro, 'Weights, vertices and a correspondence of characters in groups of odd order', Math. Zeitschrifi 212 (1993), 535-544.

18. T. Okuyama, 'Vertices of irreducible modules of p-solvable groups', (undated typescript).

19. T. Okuyama, 'Module correspondences in finite groups', Hokkaido Math. J. 10 (1981), 299-318.

20. C. Picaronny and L. Puig, 'Quelques remarques sur un thème de Knörr', J. Algebra 109 (1987) 69-73.

21. L. Puig, 'Local block theory in p-solvable groups', Proc. Symp. Pure Math. 37 (1980), 385-388.

22. L. Puig, 'Pointed groups and construction of characters', Math. Zeitschrift 176 (1981), 265-292.

23. L. Puig, 'Local extensions in endo-permutation modules split a proof of Dade's theorem', Publ. Math. Univ. Paris VII 25 (1987), 199-205.

24. L. Puig, 'Pointed groups and construction of modules', J. ALgebra 116 (1988), 7-129.

25. L. Puig, (typescript letter to Professor Watanabe, 23 November 1992).

26. L. Puig, 'On Alperin's conjecture in p-solvable groups', (manuscript 1995).

27. G. R. Robinson and R. Staszewski, 'More on Alperin's conjecture', Asterisque 181-182 (1990), 237-255. 
28. D. A. Sibley, 'Vertices, blocks, and virtual characters', J. Algebra 132 (1990), 501-507.

29. J. Thevenaz, G-algebras and modular representation theory, Clarendon Press (1995).

School of Mathematics

Cardiff University of Wales

Senghennydd Road

PO Box 926

Cardiff CF2 $4 H Y, U K$

Present address:

Department of Mathematics

Bilkent University

O6533 Bilkent

Ankara, Turkey

E-mail.barker@fen.bilkent.edu.tr. 